\title{
On a Cauchy-Nicoletti type three-point boundary value problem for linear differential equations with argument deviations
}

\author{
A. Rontó and M. Rontó
}




\title{
ON A CAUCHY-NICOLETTI TYPE THREE-POINT BOUNDARY VALUE PROBLEM FOR LINEAR DIFFERENTIAL EQUATIONS WITH ARGUMENT DEVIATIONS
}

\author{
A. RONTÓ AND M. RONTÓ
}

Received 5th November, 2009

\begin{abstract}
We obtain some results concerning the investigation of the solutions of three-point Cauchy-Nicoletti type boundary value problems for a certain class of linear functional differential equations. We show that it is useful to reduce the given problem to the parametrized two-point boundary value problem for a suitably perturbed system containing some artificially introduced parameters both in the constructed inhomogeneous two-point boundary conditions and in the modified functional differential equations.

To study the transformed parametrized two-point problem, we use a method which is based upon special type of successive approximations constructed in an analytic form. We prove the uniform convergence of these approximations to the parametrized limit function. Our technique leads to a certain system of algebraic equations with respect to the introduced parameters whose solutions provide those numerical values of the parameters that correspond to the solutions of the given three-point boundary value problem.
\end{abstract}

2000 Mathematics Subject Classification: 34B15

Keywords: Functional differential equation, special deviation of argument, linear three-point boundary value problem, successive approximations

\section{INTRODUCTION}

In studies of solutions of various boundary value problems for ordinary and functional differential equations, it is often useful to possess appropriate techniques based upon some types of successive approximations constructed in an analytic form. To this class of methods belongs, in particular, the approach suggested originally in [2224] for the investigation of the periodic boundary value problems for non-autonomous systems of ordinary differential equations of the form

$$
\begin{gathered}
x^{\prime}(t)=f(t, x(t)), \quad t \in[0, T], \\
x(0)=x(T) .
\end{gathered}
$$

The research was supported in part by AS CR, Institutional Research Plan No. AV0Z10190503 (A. Rontó) and the Hungarian Scientific Research Fund through Grant No. K68311 (M. Rontó). 
Appropriate versions of the method have been obtained by various authors and can be applied in many situations for handling boundary value problems for non-linear systems of first or second order ordinary differential equations, integro-differential equations, equations with retarded argument and more general functional differential equations, as well as boundary value problems containing parameters. The given equation can be considered together with various types of boundary conditions such as the two-point conditions (both linear and non-linear), the Cauchy-Nicoletti conditions of the form

$$
x_{i}\left(t_{i}\right)=d_{i}, \quad i=1,2, \ldots, n,
$$

where $0 \leq t_{1} \leq t_{2} \leq \cdots \leq t_{n}=T$, the boundary conditions of the interpolation type

$$
x_{j}\left(t_{i}\right)=d_{i}, \quad i=1,2, \ldots, n,
$$

with $j \in\{1,2, \ldots, n\}$ and $0 \leq t_{1}<t_{2}<\cdots<t_{n}=T$, as well as with other kinds of multipoint conditions or more general functional boundary conditions.

It is clear that both the form and complexity of the given equations and boundary conditions have essential influence both on the possibility of efficient construction of approximate solutions and the solvability analysis of the given boundary value problem. We refer, e.g., to the books $[14,25,26]$, the papers $[6,7,9-12]$, and the survey [15-21] for some related references.

According to the basic idea of the method under consideration, the given boundary value problem is replaced by some "perturbed" boundary value problem containing an unknown vector-parameter $z \in \mathbb{R}^{n}$, whose value is to be determined later and which usually has the meaning of an initial value of the solution at a certain point. The solution of the modified problem is sought for in an analytic form by a suitable iteration process. The presence of a "perturbation term," which, of course, depends on the original equations and the boundary conditions, yields a system of algebraic or transcendental "determining equations," whose solutions give the numerical values of the parameter $z \in \mathbb{R}^{n}$ that correspond to the solutions of the given boundary value problem. By studying the solvability of these determining equations, one can establish existence results for the original problem.

Some modifications of the numerical-analytic approach based upon successive approximations were obtained, in particular, in [1,3-5] for the two-point boundary value problem for the system of non-linear neutral type functional differential equations

$$
\begin{gathered}
x^{\prime}(t)=f\left(t, x(\alpha(t)), x^{\prime}(\beta(t))\right), \\
A x(0)+B x(T)=d,
\end{gathered}
$$

where $\alpha, \beta:[0, T] \rightarrow[0, T]$ are given continuous functions, $A$ and $B$ are $(n \times n)$ matrices, $\operatorname{det} B \neq 0, d \in \mathbb{R}^{n}$, and the right-hand side function $f$ defined on $[0, T] \times$ $\mathbb{R}^{n} \times \mathbb{R}^{n}$ is continuous and satisfies the Lipschitz condition

$$
\left|f\left(t, u_{1}, v_{1}\right)-f\left(t, u_{2}, v_{2}\right)\right| \leq K\left|u_{1}-u_{2}\right|+L\left|v_{1}-v_{2}\right|
$$


with some non-negative constant matrices $K$ and $L$. In [8], we refined certain estimates related to the convergence analysis of successive approximations in the case of two-point linear boundary value problems for functional differential equations where the argument deviations possess certain special properties.

In this paper, our aim is to extend the techniques used in [8] to investigate solutions of a system of linear functional differential equation with the Cauchy-Nicoletti three-point boundary conditions. The difficulties related to this type of boundary conditions are due to the singularity of the matrices that determine them. To avoid dealing with singular matrices in the boundary conditions and simplify the construction of the solution in an analytic form, we use some parametrization technique on two levels. The first level allows one to replace the three-point boundary conditions by a family of parametrized two-point inhomogeneous conditions. The second level of parametrization is used for the construction and investigation of the above-mentioned perturbed system. Finally, the study of certain algebraic or transcendental determining equations gives one a possibility to obtain the numerical values of the parameters which correspond to the solutions of the given three-point boundary value problem.

\section{NOTATION}

The following notation is used in the sequel:

$C\left([0, T], \mathbb{R}^{n}\right)$ is the Banach space of the continuous functions $[0, T] \rightarrow \mathbb{R}^{n}$ with the standard uniform norm;

$L_{1}\left([0, T], \mathbb{R}^{n}\right)$ is the usual Banach space of the vector functions $[0, T] \rightarrow \mathbb{R}^{n}$ with Lebesgue integrable components;

$\mathcal{L}\left(\mathbb{R}^{n}\right)$ is the algebra of all the square matrices of dimension $n$ with real elements;

$r(Q)$ is the maximal in module eigenvalue of the matrix $Q \in \mathcal{L}\left(\mathbb{R}^{n}\right)$;

$\mathbb{1}_{m}$ is the unit matrix of dimension $m \leq n$;

$\mathbb{O}_{m, k}$ is the zero matrix of dimension $m \times k$;

$\mathbb{O}_{m}=\mathbb{O}_{m, m}$.

\section{Problem Setting}

We consider the system of $n$ linear functional differential equations of the form

$$
x^{\prime}(t)=P_{0}(t) x(t)+P_{1}(t) x(\beta(t))+f(t), \quad t \in[0, T],
$$

subjected to the inhomogeneous three-point Cauchy-Nicoletti boundary conditions

$$
\begin{aligned}
x_{1}(0) & =x_{10}, & \ldots, & x_{p}(0) & =x_{p 0}, \\
x_{p+1}(\xi) & =d_{p+1}, & \ldots, & x_{p+q}(\xi) & =d_{p+q}, \\
x_{p+q+1}(T) & =d_{p+q+1}, & \ldots, & x_{n}(T) & =d_{n} .
\end{aligned}
$$

For the sake of simplicity, we restrict ourselves to the case where only one argument deviation is present. Here, we suppose that $T \in(0,+\infty)$, the elements of the matrix-valued functions $P_{j}:[0, T] \rightarrow \mathscr{L}\left(\mathbb{R}^{n}\right), j=0,1$ are Lebesgue integrable, $f \in$ 
$L_{1}\left([0, T], \mathbb{R}^{n}\right)$, and $\beta:[0, T] \rightarrow[0, T]$ is a Lebesgue measurable function, $x_{10} \in \mathbb{R}$, $\ldots, x_{p 0} \in \mathbb{R}, d_{i} \in \mathbb{R}, i=p+1, p+2, \ldots, n$.

Thus, in (3.2), one has $p$ conditions prescribed at the point $0, q$ conditions at a point $\xi, 0<\xi<T$, and $n-(p+q)$ conditions at the point $T$. Using the diagonal matrices $A, B$, and $C$ of the form

$$
\begin{aligned}
A & :=\left(\begin{array}{ccc}
\mathbb{1}_{p} & \mathbb{O}_{p, q} & \mathbb{O}_{n-(p+q), p} \\
\mathbb{O}_{q, p} & \mathbb{O}_{q} & \mathbb{O}_{n-(p+q), q} \\
\mathbb{O}_{n-(p+q), p} & \mathbb{O}_{n-(p+q), q} & \mathbb{O}_{n-(p+q)}
\end{array}\right), \\
B & :=\left(\begin{array}{ccc}
\mathbb{O}_{p} & \mathbb{O}_{p, q} & \mathbb{O}_{n-(p+q), p} \\
\mathbb{O}_{q, p} & \mathbb{1}_{q} & \mathbb{O}_{n-(p+q), q} \\
\mathbb{O}_{n-(p+q), p} & \mathbb{O}_{n-(p+q), q} & \mathbb{O}_{n-(p+q)}
\end{array}\right), \\
C & :=\left(\begin{array}{ccc}
\mathbb{O}_{p} & \mathbb{O}_{p, q} & \mathbb{O}_{n-(p+q), p} \\
\mathbb{O}_{q, p} & \mathbb{O}_{q} & \mathbb{O}_{n-(p+q), q} \\
\mathbb{O}_{n-(p+q), p} & \mathbb{O}_{n-(p+q), q} & \mathbb{1}_{n-(p+q)}
\end{array}\right),
\end{aligned}
$$

one can rewrite the boundary conditions (3.2) in the usual matrix-vector form

$$
A x(0)+B x(\xi)+C x(T)=\bar{d},
$$

where

$$
\bar{d}=\operatorname{col}\left(x_{10}, x_{20}, \ldots, x_{p 0}, d_{p+1}, d_{p+2}, \ldots, d_{p+q}, d_{p+q+1}, \ldots, d_{n}\right) .
$$

It is obvious that each of the matrices (3.3), (3.4), and (3.5) appearing in condition (3.6) is singular, which causes some difficulties for the construction of suitable successive approximations.

\section{PARAMETRIZATION OF THE THREE-POINT BOUNDARY CONDITIONS}

Besides the three-point boundary condition (3.6), we introduce into consideration the auxiliary two-point condition

$$
A x(0)+C x(T)=\overline{\bar{d}},
$$

where $\overline{\bar{d}}=\operatorname{col}\left(x_{10}, x_{20}, \ldots, x_{p 0}, 0,0, \ldots, 0, d_{p+q+1}, \ldots, d_{n}\right)$. Compared to (3.6), condition (4.1) may be regarded as a result of "freezeing" of the value of the function at the point $\xi$.

To avoid dealing with the singular matrix $C$ in (4.1), we carry out the following parametrization:

$$
\begin{aligned}
& x_{1}(T)=\lambda_{1}, \quad x_{2}(T)=\lambda_{2}, \quad \ldots, \quad x_{p}(T)=\lambda_{p}, \\
& x_{p+1}(T)=\lambda_{p+1}, \quad x_{p+2}(T)=\lambda_{p+2}, \quad \ldots, \quad x_{p+q}(T)=\lambda_{p+q}
\end{aligned}
$$

and, instead of (4.1), we use the two-point boundary conditions

$$
A x(0)+x(T)=d(\lambda)
$$


where $\lambda=\operatorname{col}\left(\lambda_{1}, \ldots, \lambda_{p}, \lambda_{p+1}, \ldots, \lambda_{p+q}\right) \in \mathbb{R}^{p+q}$ and

$$
\begin{aligned}
d(\lambda):=\operatorname{col}\left(x_{10}+\lambda_{1}, x_{20}+\lambda_{2}, \ldots, x_{p 0}+\lambda_{p}, \lambda_{p+1}, \lambda_{p+2}, \ldots,\right. \\
\left.\lambda_{p+q}, d_{p+q+1}, \ldots, d_{n}\right) .
\end{aligned}
$$

Instead of the three-point boundary value problem (3.1), (3.6), we first consider the two-point boundary value problem (3.1), (4.3).

\section{SUBSIDIARY STATEMENTS}

In the sequel, we need several auxuliary statements, many of which are related to properties of the sequence of functions $\left\{\alpha_{m}\right\}_{m=0}^{\infty} \subset C([0, T], \mathbb{R})$ defined by the recurrence relation

$$
\alpha_{m+1}(t)=\left(1-\frac{t}{T}\right) \int_{0}^{t} \alpha_{m}(s) d s+\frac{t}{T} \int_{t}^{T} \alpha_{m}(s) d s, \quad m=0,1,2, \ldots,
$$

where $\alpha_{0}(t):=1, t \in[0, T]$. In particular, we have

$$
\alpha_{1}(t)=2 t\left(1-\frac{t}{T}\right), \quad t \in[0, T]
$$

Lemma 1. Let the sequence of functions $\left\{\alpha_{m}\right\}_{m=0}^{\infty} \subset C([0, T], \mathbb{R})$ be given by formula (5.1). Then:

(1) The function $\alpha_{m}$ is symmetric with respect to the point $\frac{T}{2}$ for all $m \geq 0$, i.e.,

$$
\begin{gathered}
\alpha_{m}(t)=\alpha_{m}(T-t), \quad t \in[0, T], \\
\alpha_{m}\left(\frac{T}{2}-t\right)=\alpha_{m}\left(\frac{T}{2}+t\right), \quad t \in[0, T / 2] .
\end{gathered}
$$

(2) Sequence (5.1) can be represented alternatively as

$$
\begin{aligned}
\alpha_{m+1}(t) & =\int_{0}^{t} \alpha_{m}(s) d s+\frac{t}{T} \int_{t}^{T-t} \alpha_{m}(s) d s \\
& =\frac{t}{T} \int_{t}^{T-t} \alpha_{m}(s) d s+\left(1-\frac{t}{T}\right) \int_{T-t}^{t} \alpha_{m}(s) d s \\
& =\int_{0}^{T-t} \alpha_{m}(s) d s+\left(1-\frac{t}{T}\right) \int_{T-t}^{t} \alpha_{m}(s) d s, \quad t \in[0, T] .
\end{aligned}
$$

(3) For any $m \geq 1, \alpha_{m}(0)=\alpha_{m}(T)=0$ and $\alpha_{m}(t)>0$ for all $t \in(0, T)$.

(4) The maximal value of every $\alpha_{m}(t), m \geq 0$, is achieved at the point $T / 2$, namely,

$$
\max _{t \in[0, T]} \alpha_{m}(t)=\alpha_{m}\left(\frac{T}{2}\right)
$$


(5) For every $m \geq 1$,

$$
\alpha_{m}^{\prime}(t) \cdot \operatorname{sign}\left(t-\frac{T}{2}\right) \leq 0, \quad t \in[0, T],
$$

that is, the function $\alpha_{m}$ is increasing on $(0, T / 2)$ and decreasing on $(T / 2, T)$.

Proof. For the proof, see [6, Lemma 1] and [8, Lemma 1].

Lemma 2. For an arbitrary essentially bounded function $u:[0, T] \rightarrow \mathbb{R}$, the estimate

$$
\left|\int_{0}^{t}\left(u(\tau)-\frac{1}{T} \int_{0}^{T} u(s) d s\right) d \tau\right| \leq \frac{\alpha_{1}(t)}{2}(\underset{s \in[0, T]}{\operatorname{ess} \sup u}(s)-\underset{s \in[0, T]}{\operatorname{ess} \inf } u(s))
$$

is true for a. e. $t \in[0, T]$, where $\alpha_{1}$ is the function defined by equality (5.2).

Proof. Inequality (5.8) is established similarly to [13, Lemma 3] (see also [14, Lemma 2.3]).

Lemma 3. The sequence of functions $\alpha_{m}, m \geq 1$, given by relation (5.1) satisfies the inequalities

$$
\begin{gathered}
\alpha_{m+1}(t) \leq \frac{3 T}{10} \alpha_{m}(t), \quad m \geq 2 ; \\
\alpha_{m+1}(t) \leq \frac{10}{9}\left(\frac{3 T}{10}\right)^{m} \alpha_{1}(t), \quad m \geq 0 .
\end{gathered}
$$

Proof. Inequalities (5.9) are established by analogy to [13, Lemma 4] (see also [14, Lemma 2.4]).

Lemma 4 ([8, Lemma 3]). Let $\beta:[0, T] \rightarrow[0, T]$ be a measurable function satisfying the condition

$$
\underset{t \in[0, T]}{\operatorname{ess} \inf }(\beta(t)-t) \operatorname{sign}\left(t-\frac{T}{2}\right) \geq 0 .
$$

Then the members of the function sequence (5.1) satisfy the pointwise estimates

$$
\alpha_{m}(\beta(t)) \leq \alpha_{m}(t), \quad t \in[0, T], m=1,2, \ldots .
$$

Remark 1. If $\beta:[0, T] \rightarrow[0, T]$ is a continuous function satisfying condition (5.10) then necessarily $\beta(0)=0, \beta(T / 2)=T / 2$, and $\beta(T)=T$.

Lemma 5 ([8, Lemma 4]). If a measurable function $\beta:[0, T] \rightarrow[0, T]$ satisfies the condition

$$
k_{\beta}:=\operatorname{ess}_{t \in[0, T]} \frac{\beta(t)(T-\beta(t))}{t(T-t)}<+\infty,
$$

then

$$
\alpha_{1}(\beta(t)) \leq k_{\beta} \alpha_{1}(t), \quad t \in[0, T] .
$$


For example, the function $\beta(t)=t^{2}, t \in[0,1]$, satisfies condition (5.12) on the interval $[0,1]$, whereas the functions $\beta(t)=\frac{1}{2} t^{2}, \beta(t)=\frac{1}{2} t, \beta(t)=\sin t, t \in[0,1]$, do not satisfy it.

\section{CONVERGENCE OF SUCCESSIVE APPROXIMATIONS FOR THE CASE OF A GENERAL TYPE OF ARGUMENT DEVIATION}

To study the solution of the auxiliary two-point boundary value problem (3.1), (4.3) let us introduce the sequence of functions

$$
\begin{aligned}
x_{m+1}(t, z, \lambda):=z & +\int_{0}^{t}\left(P_{0}(s) x_{m}(s, z, \lambda)+P_{1}(s) x_{m}(\beta(s), z, \lambda)+f(s)\right) d s \\
& -\frac{t}{T} \int_{0}^{T}\left(P_{0}(s) x_{m}(s, z, \lambda)+P_{1}(s) x_{m}(\beta(s), z, \lambda)+f(s)\right) d s \\
& +\frac{t}{T}\left(d(\lambda)-\left(A+\mathbb{1}_{n}\right) z\right),
\end{aligned}
$$

where $m \geq 0, x_{0}(t, z)=z, t \in[0, T]$,

$$
z=\operatorname{col}\left(x_{10}, x_{20}, \ldots, x_{p 0}, z_{p+1}, \ldots, z_{n}\right) \in \mathbb{R}^{n},
$$

$\lambda=\operatorname{col}\left(\lambda_{1}, \ldots, \lambda_{p}, \lambda_{p+1}, \ldots, \lambda_{p+q}\right) \in \mathbb{R}^{p+q}$ is a vector parameter, and $d(\lambda)$ is given by (4.4).

Remark 2. We emphasize that the first $p$ components of the vector $z$ are fixed and coincide with the initial values appearing in the boundary conditions (3.2), while the other its components $z_{k}, p<k \leq n$, are considered as free parameters. Thus, the expression "for all $z$," which is often used in what follows, actually means "for all $z_{p+1}, \ldots, z_{n}$." We hope that no confusion will arise.

Let us establish the convergence of the sequence (6.1) for arbitrary deviation function $\beta:[0, T] \rightarrow[0, T]$.

Theorem 1. Let the elements of matrix-valued functions $P_{i}:[0, T] \rightarrow \mathscr{L}\left(\mathbb{R}^{n}\right)$, $i=0,1$, be Lebesgue integrable, $f \in L_{1}\left([0, T], \mathbb{R}^{n}\right)$ and $\beta:[0, T] \rightarrow[0, T]$ be a Lebesgue measurable function. Moreover, assume that

$$
r\left(K_{0}+K_{1}\right)<\frac{2}{T}
$$

where

$$
K_{i}:=\underset{s \in[0, T]}{\operatorname{ess} \sup }\left|P_{i}(s)\right|, \quad i=0,1 .
$$

Then:

(1) All the members of sequence (6.1) are absolutely continuous functions satisfying the two-point boundary conditions

$$
A x_{m}(0, z, \lambda)+x_{m}(T, z, \lambda)=d(\lambda), \quad m=1,2, \ldots,
$$

for all $\lambda \in \mathbb{R}^{p+q}$ and $z$ ofform (6.2). 
(2) The sequence of functions (6.1) converges to a limit function $x^{*}(\cdot, z, \lambda)$,

$$
x^{*}(t, z, \lambda)=\lim _{m \rightarrow \infty} x_{m}(t, z, \lambda)
$$

uniformly in $t \in[0, T]$ for all fixed $z$ of form (6.2) and $\lambda \in \mathbb{R}^{p+q}$.

(3) The limit function (6.6) satisfies the initial condition

$$
x^{*}(0, z, \lambda)=z
$$

and the boundary condition (4.3)

$$
A x^{*}(0, z, \lambda)+x^{*}(T, z, \lambda)=d(\lambda)
$$

for all $z$ of form (6.2) and $\lambda \in \mathbb{R}^{p+q}$.

(4) For all fixed $z$ of form (6.2) and $\lambda \in \mathbb{R}^{p+q}$, the limit function (6.6) is a unique absolutely continuous solution of the integro-functional equation

$$
\begin{aligned}
x(t)=z & +\int_{0}^{t}\left[P_{0}(s) x(s)+P_{1}(s) x(\beta(s))+f(s)\right] d s \\
& -\frac{t}{T} \int_{0}^{T}\left[P_{0}(s) x(s)+P_{1}(s) x(\beta(s))+f(s)\right] d s \\
& +\frac{t}{T}\left[d(\lambda)-\left(A+\mathbb{1}_{n}\right) z\right], \quad t \in[0, T] .
\end{aligned}
$$

(5) The following estimate holds for all fixed $z$ of form (6.2) and $\lambda \in \mathbb{R}^{p+q}$ :

$$
\begin{aligned}
& \left|x^{*}(t, z, \lambda)-x_{m}(t, z, \lambda)\right| \leq \\
& \quad \leq\left(\frac{T}{2}\right)^{m-1}\left(K_{0}+K_{1}\right)^{m}\left(\frac{2}{T} \mathbb{1}_{n}-K_{0}-K_{1}\right)^{-1} \gamma(z, \lambda),
\end{aligned}
$$

where

$$
\gamma(z, \lambda):=\frac{T}{2} \delta(z)+\left|d(\lambda)-\left(A+\mathbb{1}_{n}\right) z\right|
$$

and

$$
\begin{aligned}
& \delta(z):=\frac{1}{2}\left(\operatorname{ess}_{s \in[0, T]}\left(P_{0}(s) z+P_{1}(s) z+f(s)\right)\right. \\
& \left.-\underset{s \in[0, T]}{\operatorname{essinf}}\left(P_{0}(s)+P_{1}(s)+f(s)\right)\right) .
\end{aligned}
$$

In (6.10) and similar relations, below the signs $|\cdot|, \leq, \geq$, ess sup, and ess inf are understood componentwise.

Proof. The validity of assertion 1 is verified by direct computation. To obtain the other required properties, let us show that, under the conditions assumed, sequence (6.1) is a Cauchy sequence in the Banach space $C\left([0, T], \mathbb{R}^{n}\right)$ equipped with the 
standard uniform norm. Indeed, due to estimate (5.8) of Lemma 2, it follows from (6.1) that, for $m=0$ and arbitrary fixed $z_{p+1}, \ldots, z_{n}$ and $\lambda \in \mathbb{R}^{p+q}$,

$$
\begin{aligned}
\left|x_{1}(t, z, \lambda)-z\right|=\mid \int_{0}^{t} & \left(\left(P_{0}(s) z+P_{1}(s) z+f(s)\right)\right. \\
& \left.-\frac{1}{T} \int_{0}^{T}\left(P_{0}(\tau) z+P_{1}(\tau) z+f(\tau)\right) d \tau\right) d s \\
& +\frac{t}{T}\left[d(\lambda)-\left(A+\mathbb{1}_{n}\right) z\right] \mid \leq \alpha_{1}(t) \delta(z)+\tilde{\delta}(z, \lambda),
\end{aligned}
$$

where the vector $z$ has form (6.2), $\tilde{\delta}$ is defined by the formula

$$
\tilde{\delta}(z, \lambda):=\left|d(\lambda)-\left(A+\mathbb{1}_{n}\right) z\right|,
$$

and $\alpha_{1}$ is the function given by (5.2).

Let us put

$$
r_{m+1}(t, z, \lambda):=x_{m+1}(t, z, \lambda)-x_{m}(t, z, \lambda) .
$$

Then, by virtue of formulae (6.1), for all $t \in[0, T], n \geq 1, \lambda \in \mathbb{R}^{p+q}$, and $z$, we have

$$
\begin{aligned}
r_{m+1}(t, z, \lambda)= & \int_{0}^{t}\left[P_{0}(s) r_{m}(s, z, \lambda)+P_{1}(s) r_{m}(\beta(s), z, \lambda)\right] d s \\
& -\frac{t}{T} \int_{0}^{T}\left[P_{0}(s) r_{m}(s, z, \lambda)+P_{1}(s) r_{m}(\beta(s), z, \lambda)\right] d s \\
= & \left(1-\frac{t}{T}\right) \int_{0}^{t}\left[P_{0}(s) r_{m}(s, z, \lambda)+P_{1}(s) r_{m}(\beta(s), z, \lambda)\right] d s \\
& -\frac{t}{T} \int_{t}^{T}\left[P_{0}(s) r_{m}(s, z, \lambda)+P_{1}(s) r_{m}(\beta(s), z, \lambda)\right] d s .
\end{aligned}
$$

Equalities (6.16) imply that, for all $t \in[0, T], m=1,2, . ., \lambda$, and $z$,

$$
\begin{gathered}
\left|r_{m+1}(t, z, \lambda)\right| \leq K_{0}\left(\left(1-\frac{t}{T}\right) \int_{0}^{t}\left|r_{m}(s, z, \lambda)\right| d s+\frac{t}{T} \int_{t}^{T}\left|r_{m}(s, z, \lambda)\right| d s\right) \\
\quad+K_{1}\left(\left(1-\frac{t}{T}\right) \int_{0}^{t}\left|r_{m}(\beta(s), z, \lambda)\right| d s+\frac{t}{T} \int_{t}^{T} r_{m}(\beta(s), z, \lambda) d s\right),
\end{gathered}
$$

where $K_{0}$ and $K_{1}$ are the non-negative matrices given by formula (6.4). Relation (6.13) yields

$$
\left|r_{1}(t, z, \lambda)\right| \leq \alpha_{1}(t) \delta(z)+\tilde{\delta}(z, \lambda), \quad t \in[0, T],
$$

where $\delta$ and $\tilde{\delta}$ are given by (6.12) and (6.14). In view of property (5.6) of Lemma 1 , estimate (6.18) gives

$$
\left|r_{1}(t, z, \lambda)\right| \leq \frac{T}{2} \delta(z)+\tilde{\delta}(z, \lambda)=\gamma(z, \lambda), \quad t \in[0, T] .
$$


Let us now estimate $\left|r_{2}(t, z, \lambda)\right|$ using (6.17) and (6.19). We obtain

$$
\begin{aligned}
\left|r_{2}(t, z, \lambda)\right| \leq & K_{0}\left(\left(1-\frac{t}{T}\right) \int_{0}^{t}\left|r_{1}(s, z, \lambda)\right| d s+\frac{t}{T} \int_{t}^{T}\left|r_{1}(s, z, \lambda)\right| d s\right) \\
& +K_{1}\left(\left(1-\frac{t}{T}\right) \int_{0}^{t}\left|r_{1}(\beta(s), z, \lambda)\right| d s+\frac{t}{T} \int_{t}^{T}\left|r_{1}(\beta(s), z, \lambda)\right| d s\right) \\
\leq & K_{0}\left(\left(1-\frac{t}{T}\right) \int_{0}^{t} \gamma(z, \lambda) d s+\frac{t}{T} \int_{t}^{T} \gamma(z, \lambda) d s\right) \\
& +K_{1}\left(\left(1-\frac{t}{T}\right) \int_{0}^{t} \gamma(z, \lambda) d s+\frac{t}{T} \int_{t}^{T} \gamma(z, \lambda) d s\right)
\end{aligned}
$$

for all $t \in[0, T]$. Taking relations (5.1), (5.2) into account and using property (5.6), from (6.20) we get

$$
\max _{t \in[0, T]}\left|r_{2}(t, z, \lambda)\right| \leq\left(K_{0}+K_{1}\right) \gamma(z, \lambda) \max _{t \in[0, T]} \alpha_{1}(t)=\frac{T}{2}\left(K_{0}+K_{1}\right) \gamma(z, \lambda) .
$$

Arguing by induction, we then obtain that, for all $t \in[0, T]$ and $m=1,2, \ldots$,

$$
\left|r_{m}(t, z, \lambda)\right| \leq\left(\frac{T}{2}\right)^{m-1}\left(K_{0}+K_{1}\right)^{m-1} \gamma(z, \lambda)=G^{m-1} \gamma(z, \lambda),
$$

where

$$
G:=\frac{T}{2}\left(K_{0}+K_{1}\right)
$$

Due to equality (6.15), estimate (6.21), assumption (6.3), and notation (6.22) give

$$
\begin{aligned}
\left|x_{m+j}(t, z, \lambda)-x_{m}(t, z, \lambda)\right| & \leq \sum_{i=1}^{j}\left|r_{m+i}(t, z, \lambda)\right| \leq G^{m} \sum_{i=0}^{j-1} G^{i} \gamma(z, \lambda) \\
& \leq G^{m} \sum_{i=0}^{\infty} G^{i} \gamma(z, \lambda) \\
& =G^{m}\left(\mathbb{1}_{n}-G\right)^{-1} \gamma(z, \lambda)
\end{aligned}
$$

for all $t \in[0, T], m=1,2, \ldots$

Since, due to (6.3), $\lim _{m \rightarrow \infty} G^{m}=\mathbb{D}_{n}$, it is clear from (6.23) that (6.1) is a Cauchy sequence in the Banach space $C\left([0, T], \mathbb{R}^{n}\right)$ and, consequently, it converges uniformly in $t \in[0, T]$ for all fixed $z$ of form (6.2) and $\lambda \in \mathbb{R}^{p+1}$, i. e., assertion 2 holds. Assertions $2-5$ are obtained by passing to the limit.

Passing to the limit as $m \rightarrow \infty$ in (6.1) and (6.5), we show that function (6.6) is a solution of equation (6.9) and possesses property (6.8). Passing to the limit as $j \rightarrow \infty$ 
A CAUCHY-NICOLETTI TYPE PROBLEM FOR EQUATIONS WITH ARGUMENT DEVIATIONS 183

in (6.23), we obtain the estimate

$$
\left|x^{*}(t, z, \lambda)-x_{m}(t, z, \lambda)\right| \leq G^{m}\left(\mathbb{1}_{n}-G\right)^{-1} \gamma(z, \lambda)
$$

for all $t \in[0, T], m=1,2, \ldots, \lambda \in \mathbb{R}^{p+q}$, and $z$ of form (6.2), i. e., assertion 5 holds. This completes the proof of Theorem 1 .

Remark 3. A similar scheme can be obtained if the recurrence formula (6.1) is replaced (cf. [9]) by the relation

$$
\begin{array}{r}
x_{m+1}(t, z, \lambda):=z+\int_{0}^{t}\left[P_{0}(s) x_{m}(s, z, \lambda)+P_{1}(s) x_{m}(\beta(s), z, \lambda)+f(s)\right] d s \\
-\frac{\omega(t)}{T} \int_{0}^{T}\left[P_{0}(s) x_{m}(s, z, \lambda)+P_{1}(s) x_{m}(\beta(s), z, \lambda)+f(s)\right] d s \\
+\frac{\omega(t)}{T}\left[d(\lambda)-\left(A+\mathbb{1}_{n}\right) z\right],
\end{array}
$$

where $\omega:[0, T] \rightarrow[0, T]$ is an arbitrary continuous function with the properties $\omega(0)=0$ and $\omega(T)=T$.

Proposition 1. If, under assumptions of Theorem 1 , the function $x^{*}(\cdot, z, \lambda)$ satisfies the condition

$$
\begin{array}{r}
d(\lambda)-\left(A+\mathbb{1}_{n}\right) z=\int_{0}^{T}\left(P_{0}(s) x^{*}(s, z, \lambda)+P_{1}(s) x^{*}(\beta(s), z, \lambda)\right) d s \\
+\int_{0}^{T} f(s) d s
\end{array}
$$

for certain values of $z$ and $\lambda$, then, for these $z$ and $\lambda$, it is also a solution of the boundary value problem (3.1), (4.3).

The proof of the last statement is a straightforward application of the above theorem.

\section{Properties of THE LIMIT FUnCTION}

Let us first establish a relation between the limit function of sequence (6.1) and the solution of the auxiliary two-point parametrized boundary value problem (3.1), (4.3). Along with system (3.1), we also consider the system with the additive perturbation of the right-hand side

$$
x^{\prime}(t)=P_{0}(t) x(t)+P_{1}(t) x(\beta(t))+f(t)+\mu, \quad t \in[0, T],
$$

with the initial condition

$$
x(0)=z,
$$

where $\mu=\operatorname{col}\left(\mu_{1}, \ldots, \mu_{n}\right)$ is a control parameter. We shall see that, for any $z$, the vector-parameter $\mu$ can always be chosen so that the solution $x(\cdot, z, \mu)$ of the initial 
value problem (7.1), (7.2) is, at the same time, a solution of the two-point boundary value problem (7.1), (4.3).

Proposition 2. Assume that the system of differential equations (3.1) satisfies the conditions of Theorem 1. Then, for arbitrary $z$ of form (6.2) and any $\lambda$,

$$
\begin{aligned}
\mu=\frac{1}{T}[d(\lambda) & \left.-\left(A+\mathbb{1}_{n}\right) z\right] \\
& -\frac{1}{T} \int_{0}^{T}\left(P_{0}(s) x^{*}(s, z, \lambda)+P_{1}(s) x^{*}(\beta(s), z, \lambda)+f(s)\right) d s
\end{aligned}
$$

is the unique value of the vector parameter $\mu$ for which the solution $x(\cdot, z, \mu)$ of the initial value problem (7.1), (7.2) with $\mu$ given by (7.3) is also a solution of the boundary value problem (7.1), (4.3). Moreover, with this values of $\mu$

$$
x(t, z, \mu)=x^{*}(t, z, \lambda)=\lim _{m \rightarrow \infty} x_{m}(t, z, \lambda),
$$

where $\left\{x_{m}(\cdot, z, \lambda)\right\}_{m=1}^{\infty}$ is the sequence of functions defined according to (6.1).

Proof. The assertion of Proposition 2 is obtained by analogy to the proof of [11, Theorem 4.2]

Definition 1. For any $k=1,2, \ldots, n$, let us define the $n$-dimensional row vector $e_{k}$ by putting

$$
e_{k}:=\underbrace{(0,0, \ldots, 0,0,1}_{k-1}, 0, \ldots, 0) .
$$

Let us consider the function $\Delta: \mathbb{R}^{n-p} \times \mathbb{R}^{p+q} \rightarrow \mathbb{R}^{n}$ given by the formula

$$
\begin{aligned}
\Delta(z, \lambda):= & \frac{1}{T}\left[d(\lambda)-\left(A+\mathbb{1}_{n}\right) z\right]- \\
& -\frac{1}{T} \int_{0}^{T}\left[P_{0}(s) x^{*}(s, z, \lambda)+P_{1}(s) x^{*}(\beta(s), z, \lambda)+f(s)\right] d s
\end{aligned}
$$

for $z$ of form (6.2) with arbitrary $z_{p+1}, \ldots, z_{n}$ and $\lambda \in \mathbb{R}^{p+q}$. Formula (7.6) makes sense provided that the limit function $x^{*}(\cdot, z, \lambda)$ of sequence (6.1) exists.

Proposition 3. Assume the conditions of Theorem 1. Then the function $x^{*}(\cdot, z, \lambda)$ is a solution of the three-point Cauchy-Nicoletti boundary value problem (3.1), (3.6) if and only if the pair $(z, \lambda)$ satisfies the system of $n+q$ algebraic equations ${ }^{*}$

$$
\begin{aligned}
\Delta(z, \lambda) & =0, \\
e_{p+1} x^{*}(\xi, z, \lambda)=d_{p+1}, \quad \ldots, & e_{p+q} x^{*}(\xi, z, \lambda)=d_{p+q} .
\end{aligned}
$$

\footnotetext{
${ }^{*}$ Recall that the first $p$ components of $z$ are known, see Remark 2.
} 
Proof. It is sufficient to apply Proposition 2 and notice that the differential equation obtained by the differentition of equation (6.9) coincides with (3.1) if and only if the pair $(z, \lambda)$ satisfies (7.7). On the other hand, equations (7.8) bring us from the auxiliary boundary conditions (4.1) back to the three-point Cauchy-Nicoletti conditions (3.6).

Proposition 4. Let us define the matrix $R$ by putting

$$
R:=\sup _{t \in[0, T]}\left|\mathbb{1}_{n}-t T^{-1}\left(\mathbb{1}_{n}+A\right)\right| .
$$

Under the conditions of Theorem 1, the estimate

$$
\left|x^{*}\left(t, z^{0}, \lambda\right)-x^{*}\left(t, z^{1}, \lambda\right)\right| \leq \frac{2}{T}\left(\frac{2}{T} \mathbb{1}_{n}-K_{0}-K_{1}\right)^{-1} R\left|z^{0}-z^{1}\right|,
$$

where

$$
z^{j}=\operatorname{col}\left(x_{10}, x_{20}, \ldots, x_{p 0}, z_{p+1}^{j}, z_{p+2}^{j}, \ldots, z_{n}^{j}\right), \quad j=0,1,
$$

holds for arbitrary $z_{k}^{j}, k=p+1, p+2, \ldots, n, j=0,1, t \in[0, T]$, and $\lambda \in \mathbb{R}^{p+q}$.

Proof. Consider the sequence of vectors $c_{m}, m \geq 1$, defined by the recurrence formula

$$
c_{m}:=R\left|z^{0}-z^{1}\right|+\frac{T}{2}\left(K_{0}+K_{1}\right) c_{m-1}, \quad m \geq 1,
$$

with $c_{0}:=\left|z^{0}-z^{1}\right|$ and the matrix $R$ of form (7.9). Let us show that the functions

$$
u_{m}(t):=x_{m}\left(t, z^{0}, \lambda\right)-x_{m}\left(t, z^{1}, \lambda\right), \quad t \in[0, T], m \geq 1,
$$

satisfy the estimate

$$
\left|u_{m}(t)\right| \leq c_{m}, \quad t \in[0, T], m \geq 1 .
$$

Indeed, for $m=0$ relation (7.13) is satisfied in the form of an equality. Assume that (7.13) is satisfied for a given $m \geq 1$. It follows immediately from (6.1) that

$$
\begin{aligned}
u_{m+1}(t)= & z^{0}-z^{1}-\frac{t}{T}\left(\mathbb{1}_{n}+A\right)\left(z^{0}-z^{1}\right) \\
& +\int_{0}^{t}\left(P_{0}(s) u_{m}(s)+P_{1}(s) u_{m}(\beta(s))\right) d s \\
& -\frac{t}{T} \int_{0}^{T}\left(P_{0}(s) u_{m}(s)+P_{1}(s) u_{m}(\beta(s))\right) d s \\
= & z^{0}-z^{1}-\frac{t}{T}\left(\mathbb{1}_{n}+A\right)\left(z^{0}-z^{1}\right) \\
& +\left(1-\frac{t}{T}\right) \int_{0}^{t}\left(P_{0}(s) u_{m}(s)+P_{1}(s) u_{m}(\beta(s))\right) d s
\end{aligned}
$$




$$
-\frac{t}{T} \int_{t}^{T}\left(P_{0}(s) u_{m}(s)+P_{1}(s) u_{m}(\beta(s))\right) d s,
$$

whence, in view of (7.9),

$$
\begin{aligned}
\left|u_{m+1}(t)\right| \leq & R\left|z^{0}-z^{1}\right| \\
& +\left(1-\frac{t}{T}\right) \int_{0}^{t}\left(\left|P_{0}(s)\right|\left|u_{m}(s)\right|+\left|P_{1}(s)\right|\left|u_{m}(\beta(s))\right|\right) d s \\
& +\frac{t}{T} \int_{t}^{T}\left(\left|P_{0}(s)\right|\left|u_{m}(s)\right|+\left|P_{1}(s)\right|\left|u_{m}(\beta(s))\right|\right) d s
\end{aligned}
$$

for all $t \in[0, T]$ and $m \geq 1$. Recalling formulae (6.4), (5.1) and using assumption (7.13) and equality (5.6), we obtain

$$
\begin{aligned}
\left|u_{m+1}(t)\right| \leq & R\left|z^{0}-z^{1}\right|+\left(1-\frac{t}{T}\right) \int_{0}^{t}\left(K_{0}\left|u_{m}(s)\right|+K_{1}\left|u_{m}(\beta(s))\right|\right) d s \\
& +\frac{t}{T} \int_{t}^{T}\left(K_{0}\left|u_{m}(s)\right|+K_{1}\left|u_{m}(\beta(s))\right|\right) d s \\
\leq & R\left|z^{0}-z^{1}\right|+\left(\left(1-\frac{t}{T}\right) \int_{0}^{t} d t+\int_{t}^{T} d t\right)\left(K_{0}+K_{1}\right) c_{m} \\
\leq & R\left|z^{0}-z^{1}\right|+\alpha_{1}\left(\frac{T}{2}\right)\left(K_{0}+K_{1}\right) c_{m} \\
= & R\left|z^{0}-z^{1}\right|+\frac{T}{2}\left(K_{0}+K_{1}\right) c_{m}=c_{m+1},
\end{aligned}
$$

that is, estimate (7.13) holds at the step $m+1$, and, hence, it is satisfied on every step of iteration. Considering now inequality (7.13) and iterating backwards, we obtain

$$
\begin{aligned}
\left|u_{m}(t)\right| & \leq R\left|z^{0}-z^{1}\right|+\frac{T}{2}\left(K_{0}+K_{1}\right) c_{m-1} \\
& =R\left|z^{0}-z^{1}\right|+\frac{T}{2}\left(K_{0}+K_{1}\right)\left(R\left|z^{0}-z^{1}\right|+\frac{T}{2}\left(K_{0}+K_{1}\right) c_{m-2}\right) \\
& =\left(\mathbb{1}_{n}+\frac{T}{2}\left(K_{0}+K_{1}\right)\right) R\left|z^{0}-z^{1}\right|+\left(\frac{T}{2}\right)^{2}\left(K_{0}+K_{1}\right)^{2} c_{m-2}
\end{aligned}
$$

and so on, which leads us the inequality

$$
\left|u_{m}(t)\right| \leq \sum_{i=0}^{m-1}\left(\frac{T}{2}\right)^{i}\left(K_{0}+K_{1}\right)^{i} R\left|z^{0}-z^{1}\right|+\left(\frac{T}{2}\right)^{m}\left(K_{0}+K_{1}\right)^{m}\left|z^{0}-z^{1}\right|
$$


valid for all $m=1,2, \ldots$ and $t \in[0, T]$. Passing to the limit as $m \rightarrow \infty$, using assumption (6.3), and recalling notation (7.12), we obtain the inequality

$$
\left|x^{*}\left(t, z^{0}, \lambda\right)-x^{*}\left(t, z^{1}, \lambda\right)\right| \leq \sum_{i=0}^{\infty}\left(\frac{T}{2}\right)^{i}\left(K_{0}+K_{1}\right)^{i} R\left|z^{0}-z^{1}\right|, \quad t \in[0, T],
$$

whence the required estimate (7.10) follows.

Let us put

$$
\varsigma_{\theta}:=\left(K_{0}+K_{1}\right)\left(\theta \mathbb{1}_{n}-K_{0}-K_{1}\right)^{-1}
$$

for all those $\theta$ for which the inverse matrix exists.

Proposition 5. Under the conditions of Theorem 1, formula (7.6) determines a well-defined function $\Delta: \mathbb{R}^{n-p} \times \mathbb{R}^{p+q} \rightarrow \mathbb{R}^{n}$, which satisfies the estimate

$$
\left|\Delta\left(z^{0}, \lambda\right)-\Delta\left(z^{1}, \lambda\right)\right| \leq\left(\frac{1}{T}\left|A+\mathbb{1}_{n}\right|+\frac{2}{T} \oint_{\frac{2}{T}} R\right)\left|z^{0}-z^{1}\right|,
$$

where the matrices $S_{2 T^{-1}}$ and $R$ are given by (7.17) and (7.9).

For the proof of the last statement, it is sufficient to recall formula (7.6) and use Proposition 3.

\section{AN EXISTENCE THEOREM FOR THE CAUCHY-NiCOLETTI PROBLEM}

Theorem 1 and Proposition 3 give the following numerical-analytic algorithm for the construction of a solution of the three-point Cauchy-Nicoletti boundary value problem (3.1), (3.6).

(1) For any vector $z$ of form (6.2), according to (6.1), we analytically construct the sequence of functions $x_{m}(\cdot, z, \lambda)$, depending on the parameters $\left(z_{p+1}, \ldots, z_{n}\right) \in \mathbb{R}^{n-p}$ and $\lambda=\operatorname{col}\left(\lambda_{1}, \ldots, \lambda_{p}, \lambda_{p+1}, \ldots, \lambda_{p+q}\right) \in \mathbb{R}^{p+q}$ and satisfying the auxiliary two-point boundary conditions (4.3).

(2) We find the limit $x^{*}(\cdot, z, \lambda)$ of the sequence $x_{m}(\cdot, z, \lambda)$ satisfying to (4.3).

(3) We construct the algebraic determining system of the form (7.7), (7.8) with respect to the $n+q$ scalar parameters $\lambda=\operatorname{col}\left(\lambda_{1}, \ldots, \lambda_{p}, \lambda_{p+1}, \ldots, \lambda_{p+q}\right)$ and $\left(z_{p+1}, \ldots, z_{n}\right) \in \mathbb{R}^{n-p}$.

(4) Using a suitable method for the numerical solution of system (7.7), (7.8), we (approximately) find the solution

$$
\begin{gathered}
\operatorname{col}\left(z_{p+1}^{*}, \ldots, z_{n}^{*}\right) \in \mathbb{R}^{n-p}, \\
\lambda^{*}=\operatorname{col}\left(\lambda_{1}^{*}, \ldots, \lambda_{p}^{*}, \lambda_{p+1}^{*}, \ldots, \lambda_{p+q}^{*}\right) \in \mathbb{R}^{p+q}
\end{gathered}
$$

of the determining system (7.7), (7.8).

(5) Substituting values $(8.1)$ into $x^{*}(\cdot, z, \lambda)$, we get the solution of the three-point Cauchy-Nicoletti boundary value problem (3.1), (3.6) in the form

$$
x=x^{*}\left(\cdot, z^{*}, \lambda^{*}\right),
$$


where $z^{*}=\operatorname{col}\left(x_{10}, x_{20}, \ldots, x_{p 0}, z_{p+1}^{*}, \ldots, z_{n}^{*}\right)$. The solution (8.2) can also be obtained by solving the Cauchy problem

$$
x(0)=z^{*}
$$

for equation (3.1).

A fundamental difficulty in the realization of this approach is related to the analytic construction of the limit function $x^{*}(\cdot, z, \lambda)$. However, in a number of cases, this problem can be avoided because, as can be shown, it is possible to prove the existence of a solution of the three-point Cauchy-Nicoletti boundary value problem (3.1), (3.6) based on properties of a certain approximation $x_{m}(\cdot, z, \lambda)$ known in the analytic form.

Given some $m \geq 1$, define the function $\Delta_{m}: \mathbb{R}^{n} \times \mathbb{R}^{p+q} \rightarrow \mathbb{R}^{n}$ according to the formula

$$
\begin{aligned}
\Delta_{m}(z, \lambda):= & \frac{1}{T}\left[d(\lambda)-\left(A+\mathbb{1}_{n}\right) z\right] \\
& -\frac{1}{T} \int_{0}^{T}\left[P_{0}(s) x_{m}(s, z, \lambda)+P_{1}(s) x_{m}(\beta(s), z, \lambda)+f(s)\right] d s
\end{aligned}
$$

for $z$ of form (6.2) with arbitrary $z_{p+1}, \ldots, z_{n}$, and $\lambda \in \mathbb{R}^{p+q}$. To investigate the solvability of the three-point Cauchy-Nicoletti boundary value problem (3.1), (3.6), in addition to determining system (7.7), (7.8), we introduce the $m$ th approximate determining system

$$
\begin{gathered}
\Delta_{m}(z, \lambda)=0, \\
e_{p+1} x_{m}(t, z, \lambda)(\xi)=d_{p+1}, \quad \ldots, \quad e_{p+q} x_{m}(t, z, \lambda)(\xi)=d_{p+q},
\end{gathered}
$$

where $e_{i}, i=1,2, \ldots, n$, are the vectors given by (7.5) and the vector function $x_{m}(\cdot, z, \lambda)$ is defined by formula (6.1). It is natural to expect that, under suitable conditions, systems (7.7) and (8.5) are "close enough" to one another for $m$ sufficiently large.

Lemma 6. Assume the conditions of Theorem 1. Then, for arbitrary $m \geq 1, \lambda \in$ $\mathbb{R}^{p+q}$, and all $z$ of form (6.2), the estimate

$$
\left|\Delta(z, \lambda)-\Delta_{m}(z, \lambda)\right| \leq\left(\frac{T}{2}\right)^{m-1}\left(K_{0}+K_{1}\right)^{m} \delta_{\frac{2}{T}} \gamma(z, \lambda),
$$

holds, where $\gamma(z, \lambda)$ is given by (6.11).

Proof. Indeed, let us fix arbitrary $z$ and $\lambda$ and put

$$
\psi_{m}(t):=x^{*}(t, z, \lambda)-x_{m}(t, z, \lambda), \quad t \in[0, T], m \geq 1 .
$$

By virtue of estimate (6.10) and notation (7.17), we have

$$
\left|\psi_{m}(t)\right| \leq\left(\frac{T}{2}\right)^{m-1}\left(K_{0}+K_{1}\right)^{m}\left(\frac{2}{T} \mathbb{1}_{n}-K_{0}-K_{1}\right)^{-1} \gamma(z, \lambda)
$$


A CAUCHY-NICOLETTI TYPE PROBLEM FOR EQUATIONS WITH ARGUMENT DEVIATIONS 189

$$
=\left(\frac{T}{2}\right)^{m-1}\left(K_{0}+K_{1}\right)^{m-1} \delta_{\frac{2}{T}} \gamma(z, \lambda)
$$

Therefore, according to (7.6) and (8.4),

$$
\begin{aligned}
\left|\Delta(z, \lambda)-\Delta_{m}(z, \lambda)\right| & =\frac{1}{T}\left|\int_{0}^{T}\left[P_{0}(s) \psi_{m}(s)+P_{1}(s) \psi_{m}(\beta(s))\right] d s\right| \\
& \leq \frac{1}{T} \int_{0}^{T}\left(\left|P_{0}(s)\right|\left|\psi_{m}(s)\right|+\left|P_{1}(s)\right|\left|\psi_{m}(\beta(s))\right|\right) d s \\
& \leq\left(K_{0}+K_{1}\right)\left(\frac{T}{2}\right)^{m-1}\left(K_{0}+K_{1}\right)^{m-1} \delta_{\frac{2}{T}} \gamma(z, \lambda) \\
& =\left(\frac{T}{2}\right)^{m-1}\left(K_{0}+K_{1}\right)^{m} \delta_{\frac{2}{T}} \gamma(z, \lambda),
\end{aligned}
$$

which leads us to (8.6).

Let us formulate a statement that gives sufficient conditions for the solvability of the three-point Cauchy-Nicoletti boundary value problem (3.1), (3.6).

Definition 2. For any indices $i_{1}$ and $i_{2}$ between 1 and $n, i_{2} \geq i_{1}$, define the $\left(i_{2}-\right.$ $\left.i_{1}\right) \times n$ matrix $J_{i_{1}, i_{2}}$ by putting

$$
J_{i_{1}, i_{2}}:=\left(\begin{array}{lll}
\mathbb{O}_{i_{2}-i_{1}+1, i_{1}-1} & \mathbb{1}_{i_{2}-i_{1}+1} & \mathbb{O}_{i_{2}-i_{1}+1, n-i_{2}}
\end{array}\right),
$$

so that the left multiplication of a vector by the matrix $J_{i_{1}, i_{2}}$ is equivalent to the selection of its components with numbers from $i_{1}$ to $i_{2}$.

Introduce the mapping $\Phi_{m}: \mathbb{R}^{n-p} \times \mathbb{R}^{p+q} \rightarrow \mathbb{R}^{n+q}$ by setting

$$
\Phi_{m}(z, \lambda):=\left(\begin{array}{c}
\Delta_{m}(z, \lambda) \\
J_{p+1, p+q} x_{m}(\xi, z, \lambda)-J_{p+1, p+q} \bar{d}
\end{array}\right)
$$

for all $z$ of form (6.2), $\lambda \in \mathbb{R}^{p+q}$, and $m \geq 0$, where $\bar{d}$ is the vector given by equality (3.7). Recall that the first $p$ components of the vector $z$ are fixed and, thus, the actual number of variables on which $\Phi_{m}$ depends is $n+q$ (see Remark 2).

Definition 3. Let $H \subset \mathbb{R}^{n+q}$ be a an arbitrary non-empty set. For any pair of functions $f_{j}=\left(f_{j, i}\right)_{i=1}^{n+q}: H \rightarrow \mathbb{R}^{n+q}, j=1,2$, we write $f_{1} \triangleright_{H} f_{2}$ if and only if there exists a function $k: H \rightarrow\{1,2, \ldots, n+q\}$ such that

$$
f_{1, k(x)}(x)>f_{2, k(x)}(x)
$$

for all $x \in H$.

Remark 4. The relation " $\nabla_{H}$ " has properties similar to those of the usual inequality. In particular, if $f_{1} \geq f_{2}$ on $H$ pointwise and componentwise and $f_{2} \triangleright_{H} f_{3}$, then $f_{1}$ and $f_{3}$ satisfy the relation $f_{1} \triangleright_{H} f_{3}$. 
Let $D \subset \mathbb{R}^{n-p}, \Lambda \subset \mathbb{R}^{p+q}$, and $\Omega \subset D \times \Lambda$ be the closure of a bounded domain. The following theorem holds.

Theorem 2. Let us suppose that, in addition to the assumptions of Theorem 1, the set $\Omega$ and a number $m \in \mathbb{N}$ can be chosen so that the approximate determining function $\Delta_{m}$ constructed according to equation (8.5) satisfies the following conditions:

(1) The relation

$$
\left|\Phi_{m}\right| \triangleright_{\partial \Omega}\left(\frac{T}{2}\right)^{m-1}\left(\begin{array}{c}
\left(K_{0}+K_{1}\right)^{m} \delta_{\frac{2}{T}} \gamma \\
J_{p+1, p+q}\left(K_{0}+K_{1}\right)^{m-1} \oint_{\frac{2}{T}} \gamma
\end{array}\right)
$$

holds, where $\gamma$ is the function defined by formula $(6.11){ }^{\dagger}$

(2) The Brouwer degree of $\Phi_{m}$ over $\Omega$ with respect to 0 satisfies the inequality

$$
\operatorname{deg}\left(\Phi_{m}, \Omega, 0\right) \neq 0 .
$$

Then the three-point Cauchy-Nicoletti boundary value problem (3.1), (3.6) has a solution $x$ with $\left(x_{p+1}(0), x_{p+2}(0), \ldots, x_{n}(0)\right)$ belonging to $D$.

Proof. Let us define the mapping $\Phi: \mathbb{R}^{n-p} \times \mathbb{R}^{p+q} \rightarrow \mathbb{R}^{n}$ by setting

$$
\Phi(z, \lambda):=\left(\begin{array}{c}
\Delta(z, \lambda) \\
J_{p+1, p+q} x^{*}(\xi, z, \lambda)-J_{p+1, p+q} \bar{d}
\end{array}\right)
$$

for all $z$ of form (6.2) and $\lambda \in \mathbb{R}^{p+q}$, where $x^{*}$ is the limit function (6.6) of sequence (6.1) and $\bar{d}$ is the vector (3.7). It is clear from from Proposition 5 that the mappings $\Phi$ and $\Phi_{m}$ are continuous.

Let us prove that the fields $\Phi$ and $\Phi_{m}$ are homotopic. For this purpose, we consider the linear deformation

$$
Q_{\theta}(\lambda):=\Phi_{m}(z, \lambda)+\theta\left[\Phi(z, \lambda)-\Phi_{m}(z, \lambda)\right], \quad(z, \lambda) \in \partial \Omega,
$$

where $\theta \in[0,1]$. Obviously, $Q_{\theta}$ is continuous mapping on $\partial \Omega$ for every $\theta \in[0,1]$ and, furthermore,

$$
Q_{0}(z, \lambda)=\Phi_{m}(z, \lambda), \quad Q_{1}(z, \lambda)=\Phi(z, \lambda)
$$

for all $(z, \lambda) \in \partial \Omega$.

For arbitrary $(z, \lambda) \in \partial \Omega$ and $\theta \in[0,1]$, in view of (8.10), (8.14), and (6.10), we have

$$
\begin{aligned}
\left|Q_{\theta}(z, \lambda)\right| & =\left|\Phi_{m}(z, \lambda)+\theta\left[\Phi(z, \lambda)-\Phi_{m}(z, \lambda)\right]\right| \\
& \geq\left|\Phi_{m}(z, \lambda)\right|-\left|\Phi(z, \lambda)-\Phi_{m}(z, \lambda)\right| .
\end{aligned}
$$

\footnotetext{
${ }^{\dagger}$ Recall that $S_{2 T^{-1}}$ is the matrix (7.17), the vector function $\gamma$ is defined by (6.11), and the matrices in (8.10) are constructed according to (8.8).
} 
On the other hand, recalling equalities (7.6), (8.4), (7.17), (8.9), and using estimates (6.10) of Theorem 1 and (8.6) of Lemma 6, we otain the componentwise inequalities

$$
\left|\Phi(z, \lambda)-\Phi_{m}(z, \lambda)\right| \leq\left(\begin{array}{c}
\left(\frac{T}{2}\right)^{m-1}\left(K_{0}+K_{1}\right)^{m-1} \delta_{\frac{2}{T}} \gamma(z, \lambda) \\
\left(\frac{T}{2}\right)^{m-1} J_{p+1, p+q}\left(K_{0}+K_{1}\right)^{m} \delta_{\frac{2}{T}} \gamma(z, \lambda)
\end{array}\right)
$$

whence, in view of (8.15), it follows that

$$
\left|Q_{\theta}\right| \triangleright_{\partial \Omega} 0, \quad \theta \in[0,1] \text {. }
$$

The last relation implies, in particular, that $Q_{\theta}$ does not vanish on $\partial \Omega$ for any value of $\theta \in[0,1]$, i. e., deformation (8.13) is non-degenerate and, thus, $\Phi_{m}$ is homotopic to $\Phi$. Using assumption (8.11) and the property of invariance of degree under homotopy, we conclude that

$$
\operatorname{deg}(\Phi, \Omega, 0)=\operatorname{deg}\left(\Phi_{m}, \Omega, 0\right) \neq 0 .
$$

The classical topological result (see, e. g., [2, Theorem A2.5]) then guarantees the existence of vectors $\left(z^{*}, \lambda^{*}\right) \in \Omega$ such that $\Phi\left(z^{*}, \lambda^{*}\right)=0$, which, according to (8.12), means that

$$
\Delta\left(z^{*}, \lambda^{*}\right)=0
$$

and, moreover, $q$ components of the vector $x^{*}\left(\xi, z^{*}, \lambda^{*}\right)$, starting from the $(p+1)$ th, coincide with the corresponding components of $\bar{d}$. Thus, the pair $\left(z^{*}, \lambda^{*}\right)$ satisfies the system of equations (7.7), (7.8). Applying now Proposition 3, we find that function (8.2) is a solution of the three-point Cauchy-Nicoletti boundary value problem (3.1), (3.6).

\section{CONVERGENCE OF SUCCESSIVE APPROXIMATIONS FOR THE SPECIAL DEVIATION FUNCTIONS}

If the deviation function $\beta:[0, T] \rightarrow[0, T]$ satisfies the condition (5.10), then the convergence condition (5.11) can be improved.

Theorem 3. Assume that the condition

$$
r\left(K_{0}+K_{1}\right)<\frac{10}{3 T} .
$$

is satisfied and, moreover, the deviation function $\beta:[0, T] \rightarrow[0, T]$ has property (5.10). Then assertions 1-4 of Theorem 1 hold. Moreover, the estimate

$$
\begin{aligned}
\left|x^{*}(t, z, \lambda)-x_{m}(t, z, \lambda)\right| & \leq \\
& \leq \frac{20}{9}\left(\frac{3 T}{10}\right)^{m-2} t\left(1-\frac{t}{T}\right)\left(K_{0}+K_{1}\right)^{m-1} S_{\frac{10}{3 T}} \gamma(z, \lambda)
\end{aligned}
$$

holds for any $m \geq 1, \lambda \in \mathbb{R}^{p+q}, t \in[0, T]$, and all fixed $z$ ofform (6.2), where $\gamma(z, \lambda)$ and $\mathcal{S}_{\frac{10}{3 T}}$ are given by (6.11) and (7.17). 
Proof. By virtue of Lemma 4, it follows from assumption (5.10) that inequalities (5.11) are true and, in particular,

$$
\alpha_{1}(\beta(t)) \leq \alpha_{1}(t), \quad t \in[0, T] .
$$

Estimating $\left|r_{2}(t, z, \lambda)\right|$ by using (6.13), (5.1), (9.2), and (5.9), we get

$$
\begin{aligned}
\left|r_{2}(t, z, \lambda)\right| \leq & K_{0}\left(\left(1-\frac{t}{T}\right) \int_{0}^{t}\left(\frac{T}{2} \delta(z)+\tilde{\delta}(z, \lambda)\right) d s\right. \\
& \left.+\frac{t}{T} \int_{t}^{T}\left(\frac{T}{2} \delta(z)+\tilde{\delta}(z, \lambda)\right) d s\right) \\
& +K_{1}\left(\left(1-\frac{t}{T}\right) \int_{0}^{t}\left(\frac{T}{2} \delta(z)+\tilde{\delta}(z, \lambda)\right) d s\right. \\
& \left.+\frac{t}{T} \int_{t}^{T}\left(\frac{T}{2} \delta(z)+\tilde{\delta}(z, \lambda)\right) d s\right) \\
\leq & \left(K_{0}+K_{1}\right) \gamma(z, \lambda) \alpha_{1}(t), \quad t \in[0, T],
\end{aligned}
$$

where $\tilde{\delta}(z, \lambda), \delta(z)$, and $\gamma(z, \lambda)$ are given by (6.14), (6.12), and (6.11), respectively. Relation (9.3), due to (9.2), yields

$$
\begin{aligned}
\left|r_{2}(\beta(t), z, \lambda)\right| & \leq\left(K_{0}+K_{1}\right) \gamma(z, \lambda) \alpha_{1}(\beta(t)) \\
& \leq\left(K_{0}+K_{1}\right) \gamma(z, \lambda) \alpha_{1}(t), \quad t \in[0, T] .
\end{aligned}
$$

Arguing by induction, we find that all the functions (6.15) admit the estimates

$$
\begin{aligned}
\left|r_{m+1}(t, z, \lambda)\right| & \leq\left(K_{0}+K_{1}\right)^{m} \gamma(z, \lambda) \alpha_{m}(t), \\
\left|r_{m+1}(\beta(t), z, \lambda)\right| & \leq\left(K_{0}+K_{1}\right)^{m} \gamma(z, \lambda) \alpha_{m}(t),
\end{aligned}
$$

for all $t \in[0, T]$ and $m \geq 1$, where the function $\alpha_{m}$ is given by (5.1).

Due to estimate (5.9) of Lemma 3, relations (9.5), (9.6) yield

$$
\begin{aligned}
\left|r_{m+1}(t, z, \lambda)\right| & \leq \frac{10}{9}\left(\frac{3 T}{10}\right)^{m-1}\left(K_{0}+K_{1}\right)^{m} \gamma(z, \lambda) \alpha_{1}(t), \\
\left|r_{m+1}(\beta(t), z, \lambda)\right| & \leq \frac{10}{9}\left(\frac{3 T}{10}\right)^{m-1}\left(K_{0}+K_{1}\right)^{m} \gamma(z, \lambda) \alpha_{1}(t),
\end{aligned}
$$

and it remains to repeat, with obvious modifications, the reasoning shown at the end of the proof of Theorem 1.

A similar improvement of the convergence condition is possible in the case where $\beta$ satisfies condition (5.12). 
Theorem 4. Assume that the deviation function $\beta:[0, T] \rightarrow[0, T]$ has property (5.12) and, moreover,

$$
r\left(K_{0}+K_{1}\right)<\frac{3}{T \eta}
$$

where

$$
\eta:=\max \left\{k_{\beta}, 1\right\}
$$

and $k_{\beta}$ is the constant appearing in (5.12). Then assertions $1-4$ of Theorem 1 are true. Moreover, the estimate

$$
\begin{aligned}
\left|x^{*}(t, z, \lambda)-x_{m}(t, z, \lambda)\right| & \leq \\
& \leq \frac{6}{T} t\left(1-\frac{t}{T}\right)\left(\frac{T \eta}{3}\right)^{m-1}\left(K_{0}+K_{1}\right)^{m-1} \delta_{\frac{3}{T \eta}} \gamma(z, \lambda)
\end{aligned}
$$

holds for any $m \geq 1, \lambda \in \mathbb{R}^{p+q}, t \in[0, T]$, and all fixed $z$ of form (6.2).

Proof. It is clear from (9.8) that $\eta \geq 1$ and, hence, according to (9.3),

$$
\begin{aligned}
\left|r_{2}(t, z, \lambda)\right| & \leq\left(K_{0}+K_{1}\right) \gamma(z, \lambda) \alpha_{1}(t) \\
& \leq \eta\left(K_{0}+K_{1}\right) \gamma(z, \lambda) \alpha_{1}(t), \quad t \in[0, T] .
\end{aligned}
$$

Due to (5.13), we have

$$
\begin{aligned}
\left|r_{2}(\beta(t), z, \lambda)\right| & \leq\left(K_{0}+K_{1}\right) \gamma(z, \lambda) \alpha_{1}(\beta(t)) \\
& \leq k_{\beta}\left(K_{0}+K_{1}\right) \gamma(z, \lambda) \alpha_{1}(t) \\
& \leq \eta\left(K_{0}+K_{1}\right) \gamma(z, \lambda) \alpha_{1}(t), \quad t \in[0, T] .
\end{aligned}
$$

Using (6.17), (9.9), (9.10) and (5.9) and carrying out calculations, we obtain

$$
\begin{aligned}
\left|r_{3}(t, z, \lambda)\right| & \leq \eta K_{0}\left(K_{0}+K_{1}\right) \gamma(z, \lambda) \alpha_{2}(t)+\eta K_{1}\left(K_{0}+K_{1}\right) \gamma(z, \lambda) \alpha_{2}(t) \\
& \leq \eta\left(K_{0}+K_{1}\right)^{2} \gamma(z, \lambda) \alpha_{2}(t) \\
& \leq \eta \frac{T \eta}{3}\left(K_{0}+K_{1}\right)^{2} \gamma(z, \lambda) \alpha_{1}(t)
\end{aligned}
$$

Therefore, in view of (9.11), (5.12), (5.13),

$$
\begin{aligned}
\left|r_{3}(\beta(t), z, \lambda)\right| & \leq \eta\left(K_{0}+K_{1}\right)^{2} \gamma(z, \lambda) \alpha_{2}(\beta(t)) \\
& \leq \eta\left(K_{0}+K_{1}\right)^{2} \gamma(z, \lambda) \frac{T}{3} \alpha_{1}(\beta(t)) \\
& \leq \eta\left(\frac{T \eta}{3}\right)\left(K_{0}+K_{1}\right)^{2} \gamma(z, \lambda) \alpha_{1}(t) .
\end{aligned}
$$

Further on, according to (6.17), (9.11), and (9.12), we find

$$
\left|r_{4}(t, z, \lambda)\right| \leq K_{0} \eta\left(\frac{T \eta}{3}\right)\left(K_{0}+K_{1}\right)^{2} \gamma(z, \lambda) \alpha_{2}(t)
$$




$$
\begin{aligned}
& +K_{1} \eta\left(\frac{T \eta}{3}\right)\left(K_{0}+K_{1}\right)^{2} \gamma(z, \lambda) \alpha_{2}(t) \\
= & \eta\left(\frac{T \eta}{3}\right)\left(K_{0}+K_{1}\right)^{3} \gamma(z, \lambda) \alpha_{2}(t) \\
\leq & \left(\frac{T \eta}{3}\right)^{2}\left(K_{0}+K_{1}\right)^{3} \gamma(z, \lambda) \alpha_{1}(t) \\
\leq & \eta\left(\frac{T \eta}{3}\right)^{2}\left(K_{0}+K_{1}\right)^{3} \gamma(z, \lambda) \alpha_{1}(t) .
\end{aligned}
$$

According to (9.13) and (5.13),

$$
\begin{aligned}
\left|r_{4}(\beta(t), z, \lambda)\right| & \leq\left(\frac{T \eta}{3}\right)^{2}\left(K_{0}+K_{1}\right)^{3} \gamma(z, \lambda) \alpha_{1}(\beta(t)) \\
& \leq \eta\left(\frac{T \eta}{3}\right)^{2}\left(K_{0}+K_{1}\right)^{3} \gamma(z, \lambda) \alpha_{1}(t)
\end{aligned}
$$

for a. e. $t \in[0, T]$. Arguing by induction, for any $t \in[0, T]$, we arrive at the estimates

$$
\begin{aligned}
\left|r_{m}(t, z, \lambda)\right| & \leq\left(\frac{T \eta}{3}\right)^{m-2}\left(K_{0}+K_{1}\right)^{m-1} \gamma(z, \lambda) \alpha_{1}(t) \\
& =\left(K_{0}+K_{1}\right) \overline{\bar{G}}^{m-2} \gamma(z, \lambda) \alpha_{1}(t) \\
& \leq \eta\left(K_{0}+K_{1}\right) \overline{\bar{G}}^{m-2} \gamma(z, \lambda) \alpha_{1}(t),
\end{aligned}
$$

where

$$
\overline{\bar{G}}:=\frac{\eta T}{3}\left(K_{0}+K_{1}\right)
$$

Using (9.15), we also get

$$
\begin{aligned}
\left|r_{m}(\beta(t), z, \lambda)\right| & \leq k_{\beta}\left(\frac{T \eta}{3}\right)^{m-2}\left(K_{0}+K_{1}\right)^{m-1} \gamma(z, \lambda) \alpha_{1}(t) \\
& \leq \eta\left(K_{0}+K_{1}\right) \overline{\bar{G}}^{m-2} \gamma(z, \lambda) \alpha_{1}(t)
\end{aligned}
$$

for a. e. $t \in[0, T]$. This yields

$$
\begin{aligned}
\left|x_{m+j}(t, z, \lambda)-x_{m}(t, z, \lambda)\right| & \leq \sum_{i=1}^{j}\left|r_{m+i}(t, z, \lambda)\right| \\
& \leq \eta\left(K_{0}+K_{1}\right) \overline{\bar{G}}^{m-1} \sum_{i=0}^{j-1} \overline{\bar{G}}^{i} \gamma(z, \lambda) \alpha_{1}(t) \\
& \leq \eta\left(K_{0}+K_{1}\right) \overline{\bar{G}}^{m-1}\left(\mathbb{1}_{n}-\overline{\bar{G}}\right)^{-1} \gamma(z, \lambda) \alpha_{1}(t)
\end{aligned}
$$


A CAUCHY-NICOLETTI TYPE PROBLEM FOR EQUATIONS WITH ARGUMENT DEVIATIONS 195

$$
=\frac{3}{T} \overline{\bar{G}}^{m}\left(\mathbb{1}_{n}-\overline{\bar{G}}\right)^{-1} \gamma(z, \lambda) \alpha_{1}(t)
$$

for any $m \geq 1, j \geq 1, t \in[0, T]$, and arbitrary $\lambda$ and $z$. The required assertion is now obtained from (9.18) again by analogy to the proof of Theorem 1.

Remark 5. It is obvious that if $\eta<3 / 2$, then the convergence condition (9.7) is sharper than inequality (6.3) used in the general case.

Remark 6. Estimates of Theorems 3 and 4 allow one to state analogues of the existence Theorem 2. The formulations are straightforward, and we omit them.

\section{A NUMERICAL EXAMPLE}

We apply techniques based on the statements of the preceding sections to the following Cauchy-Nicoletti boundary value problem

$$
x_{1}(0)=-\frac{1}{16}, \quad x_{2}\left(\frac{1}{2}\right)=\frac{1}{8}, \quad x_{3}(1)=\frac{1}{4}
$$

for the system of three equations

$$
\left.\begin{array}{l}
x_{1}^{\prime}(t)=x_{2}\left(t^{2}\right)+\frac{t}{4}-\frac{t^{2}}{4}, \\
x_{2}^{\prime}(t)=t x_{3}(t)+\frac{1}{4}-\frac{t}{4}, \\
x_{3}^{\prime}(t)=t x_{1}(t)-\frac{t^{2}}{2} x_{2}(t)+\frac{t}{16},
\end{array}\right\}
$$

considered in the closed domain determined by the inequalities

$$
\left|x_{1}\right| \leq \frac{1}{2}, \quad\left|x_{2}\right| \leq \frac{1}{2}, \quad\left|x_{3}\right| \leq \frac{1}{3} .
$$

System (10.2), of course, is a particular case of (3.1) with $T=1, f_{1}(t)=t(1-$ $t) / 4, f_{2}(t)=(1-t) / 4, f_{3}(t)=t / 16$ for $t \in[0,1]$,

$$
P_{0}(t)=\left(\begin{array}{ccc}
0 & 0 & 0 \\
0 & 0 & t \\
t & -\frac{t^{2}}{2} & 0
\end{array}\right) \quad P_{1}(t)=\left(\begin{array}{ccc}
0 & 1 & 0 \\
0 & 0 & 0 \\
0 & 0 & \frac{t}{4}
\end{array}\right)
$$

and the argument transformation

$$
\beta(t)=t^{2}, \quad t \in[0,1],
$$

whereas (10.1) has form (3.6) with

$$
A=\left(\begin{array}{lll}
1 & 0 & 0 \\
0 & 0 & 0 \\
0 & 0 & 0
\end{array}\right), \quad B=\left(\begin{array}{lll}
0 & 0 & 0 \\
0 & 1 & 0 \\
0 & 0 & 0
\end{array}\right), \quad C=\left(\begin{array}{lll}
0 & 0 & 0 \\
0 & 0 & 0 \\
0 & 0 & 1
\end{array}\right), \quad d=\left(\begin{array}{c}
-1 / 16 \\
1 / 8 \\
1 / 4
\end{array}\right)
$$


The two-point parametrized boundary conditions (4.3) have the form

$$
\left(\begin{array}{lll}
1 & 0 & 0 \\
0 & 0 & 0 \\
0 & 0 & 0
\end{array}\right) x(0)+\left(\begin{array}{lll}
1 & 0 & 0 \\
0 & 1 & 0 \\
0 & 0 & 1
\end{array}\right) x(1)=\left(\begin{array}{c}
-\frac{1}{16}+\lambda_{1} \\
\lambda_{2} \\
\frac{1}{4}
\end{array}\right) .
$$

The deviation function (10.5) satisfies condition (5.13) because, as one can verify, $k_{\beta}$ is equal to 2 in this case and, according to (9.8), we have $\eta=2$. Furthermore, as follows immediately from (10.4), matrices (6.4) are determined by the equalities

$$
K_{0}=\left(\begin{array}{ccc}
0 & 0 & 0 \\
0 & 0 & 1 \\
1 & \frac{1}{2} & 0
\end{array}\right), \quad K_{1}=\left(\begin{array}{ccc}
0 & 1 & 0 \\
0 & 0 & 0 \\
0 & 0 & \frac{1}{4}
\end{array}\right) .
$$

Therefore, $r\left(K_{0}+K_{1}\right) \approx 1.267<\frac{5}{3}$ and, hence, condition (9.7) is satisfied. By virtue of Theorem 4, the method of successive approximations is applicable to the two-point parametrized problem (10.2), (10.6).

One can verify directly that the triplet of functions

$$
\begin{aligned}
& x_{1}^{*}(t)=\frac{t^{2}}{8}-\frac{1}{16}, \\
& x_{2}^{*}(t)=\frac{t}{4}, \\
& x_{3}^{*}(t)=\frac{1}{4}
\end{aligned}
$$

is a solution of the boundary value problem (10.2), (10.1). Let us see how the approximation scheme based on Theorem 4 works in this case.

We take the starting approximation $x_{0}=\left(x_{i 0}\right)_{i=1}^{3}$ of the form

$$
x_{0}(t)=\left(\begin{array}{c}
-\frac{1}{16} \\
z_{2} \\
z_{3}
\end{array}\right)
$$

with $z_{1}=-1 / 16$, and construct the corresponding functions of the recurrence sequence (6.1). We obtain:

$$
\begin{aligned}
& x_{m+1}(t, z, \lambda)=\left(\begin{array}{c}
-\frac{1}{16} \\
z_{2} \\
z_{3}
\end{array}\right) \\
& +\left(\begin{array}{c}
t \\
\int_{0}^{t}\left(x_{2 m}\left(s^{2}\right)+\frac{s}{4}-\frac{s^{2}}{4}\right) d s-t \int_{0}^{1}\left(x_{2 m}\left(s^{2}\right)+\frac{s}{4}-\frac{s^{2}}{4}\right) d s \\
\int_{0}^{t}\left(s x_{3 m}(s)+\frac{1}{4}-\frac{s}{4}\right) d s-\frac{t}{T} \int_{0}^{1}\left(s x_{3 m}(s)+\frac{1}{4}-\frac{s}{4}\right) d s \\
\int_{0}^{t}\left(s x_{1 m}(s)-\frac{s^{2}}{2} x_{2 m}(s)+\frac{s}{16}\right) d s-t \int_{0}^{1}\left(s x_{1 m}(s)-\frac{s^{2}}{2} x_{2 m}(s)+\frac{s}{16}\right) d s
\end{array}\right)
\end{aligned}
$$


A CAUCHY-NICOLETTI TYPE PROBLEM FOR EQUATIONS WITH ARGUMENT DEVIATIONS 197

$$
+t\left(\left(\begin{array}{c}
-\frac{1}{16}+\lambda_{1} \\
\lambda_{2} \\
\frac{1}{4}
\end{array}\right)-\left(\begin{array}{lll}
2 & 0 & 0 \\
0 & 1 & 0 \\
0 & 0 & 1
\end{array}\right)\left(\begin{array}{c}
-\frac{1}{16} \\
z_{2} \\
z_{3}
\end{array}\right)\right), \quad m=0,1,2, \ldots
$$

For $m=0$, formula (10.9) gives

$$
\begin{aligned}
& x_{11}(t, z, \lambda)=-\frac{1}{16}+\frac{t^{2}}{8}-\frac{t^{3}}{12}+\frac{t}{48}+t \lambda_{1}, \\
& x_{12}(t, z, \lambda)=z_{2}+\frac{1}{8} t+\frac{1}{2} t^{2} z_{3}-\frac{1}{8} t^{2}-\frac{1}{2} t z_{3}+t \lambda_{2}-z_{2} t, \\
& x_{13}(t, z, \lambda)=z_{3}-\frac{1}{6} z_{2} t^{3}+\frac{1}{6} z_{2} t+\frac{1}{4} t-t z_{3},
\end{aligned}
$$

and the first approximate determining system

$$
\begin{aligned}
& \Delta_{1}(z, \lambda)=0, \\
& x_{12}\left(\frac{1}{2}\right)=\frac{1}{8}
\end{aligned}
$$

has the roots

$$
\begin{array}{ll}
\lambda_{1} \approx-0.020833333, & \lambda_{2} \approx 0.2500000001, \\
z_{2} \approx 0.13 \cdot 10^{-9}, & z_{3} \approx 0.25 .
\end{array}
$$

Substituting (10.11) into (10.10), we obtain the first approximation of the solution of the given three-point Cauchy-Nicoletti boundary value problem

$$
\begin{aligned}
& x_{11}(t, z, \lambda)=-0.0625+\frac{t^{2}}{8}-\frac{t^{3}}{12}+0.13 \cdot 10^{-9} t, \\
& x_{12}(t, z, \lambda)=0.13 \cdot 10^{-9}+0.25 t, \\
& x_{13}(t, z, \lambda)=0.25-0.2166666667 \cdot 10^{-10} t^{3} .
\end{aligned}
$$

The result of computation of the second iteration is

$$
\begin{aligned}
& x_{21}(t, z, \lambda)=-\frac{1}{16}+\frac{1}{10} t^{5} z_{3}-\frac{1}{40} t^{5}-\frac{1}{24} t^{3} \\
& -\frac{1}{3} t^{3} z_{2}-\frac{1}{6} t^{3} z_{3}+\frac{1}{3} t^{3} \lambda_{2}+\frac{1}{8} t^{2}+\frac{1}{3} z_{2} t+\frac{1}{15} t z_{3}+\frac{1}{240} t-\frac{1}{3} t \lambda_{2}+t \lambda_{1}, \\
& x_{22}(t, z, \lambda)=z_{2}+\frac{1}{24} t-\frac{1}{30} z_{2} t^{5}+\frac{1}{18} t^{3} z_{2}+\frac{1}{12} t^{3}-\frac{1}{3} t^{3} z_{3}+\frac{1}{2} t^{2} z_{3}-\frac{1}{8} t^{2} \\
& -\frac{46}{45} z_{2} t-\frac{1}{6} t z_{3}+t \lambda_{2},
\end{aligned}
$$

and

$$
x_{23}(t, z, \lambda)=z_{3}-\frac{1}{20} t^{5} z_{3}-\frac{1}{240} t^{5}+\frac{1}{64} t^{4}+\frac{1}{8} t^{4} z_{2}+\frac{1}{16} t^{4} z_{3}-\frac{1}{8} t^{4} \lambda_{2}+\frac{1}{144} t^{3}
$$




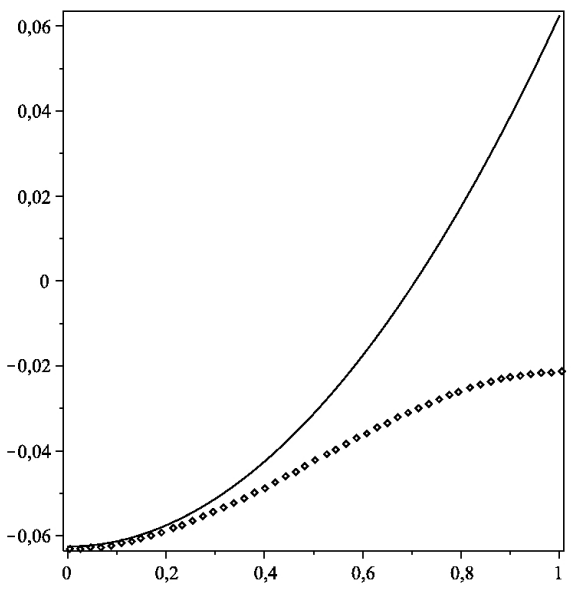

(A) First component

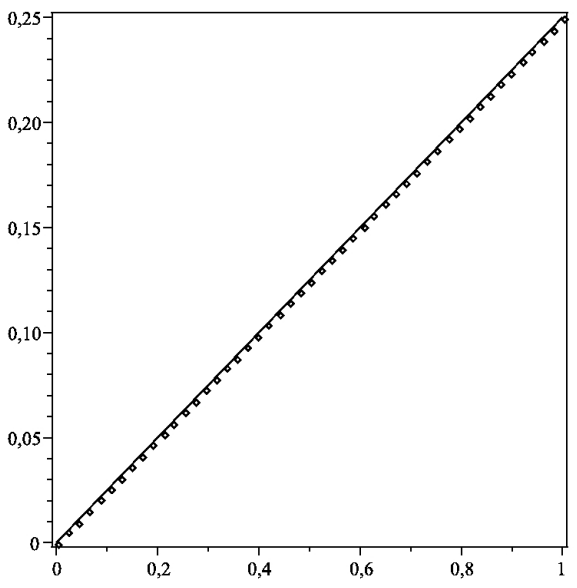

(B) Second component

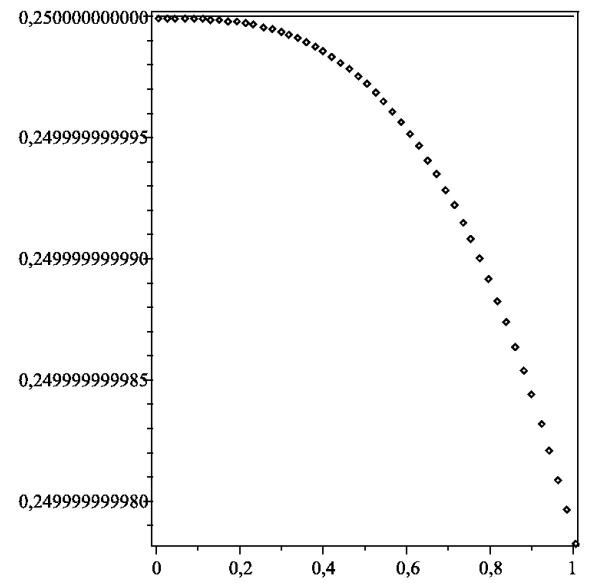

(C) Third component

FIGURE 1. The the exact solution (solid line) and its first approximation (dots).

$$
+\frac{1}{3} t^{3} \lambda_{1}-\frac{1}{16} t^{3} z_{2}-\frac{81}{80} t z_{3}+\frac{667}{2880} t+\frac{1}{24} z_{2}+\frac{1}{8} t \lambda_{2}-\frac{1}{3} t \lambda_{1} .
$$

The approximate solution of the second approximate determining equation has the form

$$
\begin{array}{ll}
\lambda_{1} \approx 0.06357861637, & \lambda_{2} \approx 0.2490649542, \\
z_{2} \approx 0.94488842 \cdot 10^{-3}, & z_{3} \approx 0.2385944095
\end{array}
$$


Inserting values (10.13) into the expressions above, we obtain the components of the second approximation which have the form

$$
\begin{aligned}
x_{21}(t)= & -0.0625-0.001140559 t^{5}+0.001274287 t^{3}+\frac{1}{8} t^{2}+0.94488842 \cdot 10^{-3} t, \\
x_{22}(t)= & 0.94488842 \cdot 10^{-3}+0.2500000001 t-0.3149628067 \cdot 10^{-4} t^{5} \\
& +0.0038543573 t^{3}-0.57027952 \cdot 10^{-2} t^{2}, \\
x_{23}(t)= & 0.2385944095-0.01609638715 t^{5}-0.4778576375 \cdot 10^{-3} t^{4} \\
& +0.02797983516 t^{3}+0.2 \cdot 10^{-9} t .
\end{aligned}
$$

Proceeding analogously, we find that the components of the fourth iteration have the form

$$
\begin{aligned}
x_{41}(t, z, \lambda)= & -\frac{1}{63} t^{7} \lambda_{1}-\frac{2167}{6552} t \lambda_{2}+\frac{1}{624} t^{13} z_{2}-\frac{1}{16}-\frac{1}{624} t^{13} \lambda_{2}+\frac{1031}{327600} t z_{3} \\
& +\frac{360259}{1081080} z_{2} t+\frac{10343}{10395} t \lambda_{1}+\frac{1}{1248} t^{13} z_{3}+\frac{1}{165} t^{11} \lambda_{1}-\frac{1}{330} t^{11} z_{2} \\
& -\frac{27}{560} t^{7} z_{3}+\frac{1}{504} t^{7} z_{2}+\frac{1}{168} t^{7} \lambda_{2}+\frac{1}{10} t^{5} z_{3}-\frac{721}{2160} t^{3} z_{2} \\
& +\frac{2}{135} t^{3} \lambda_{1}-\frac{557}{10080} t^{3} z_{3}-\frac{24869}{362880} t^{3}+\frac{1}{8} t^{2}-\frac{1}{2100} t^{15} z_{3} \\
& +\frac{2557873}{129729600} t+\frac{47}{144} t^{3} \lambda_{2}-\frac{1}{25200} t^{15}+\frac{1}{4992} t^{13}+\frac{1}{7920} t^{11} \\
& +\frac{667}{60480} t^{7}-\frac{1}{40} t^{5}, \\
x_{42}(t, z, \lambda)= & \frac{14773}{15120} t^{2} \lambda_{2}+z_{2}-\frac{1}{1728} t^{8} z_{2}-\frac{1}{48} t^{6} \lambda_{2}+\frac{23}{1080} t^{6} z_{2}+\frac{1}{288} t^{6} z_{3} \\
& -\frac{1}{45} t^{5} \lambda_{2}-\frac{1}{90} t^{5} z_{2}+\frac{1}{15} t^{5} \lambda_{1}+\frac{1}{2} t^{2} z_{3}-\frac{117}{700} t z_{3}-\frac{453277}{453600} z_{2} t \\
& -\frac{1}{2520} t^{9}-\frac{1}{1152} t^{8}+\frac{5}{1152} t^{6}+\frac{1}{4800} z_{2} t^{10}+\frac{2}{45} t \lambda_{1}-\frac{1}{84} t^{7} z_{3} \\
& -\frac{1}{105} t^{7} z_{2}+\frac{1}{105} t^{7} \lambda_{2}+\frac{1}{225} t^{5} z_{3}-\frac{13}{12960} t^{3} z_{2}-\frac{1}{9} t^{3} \lambda_{1}-\frac{187}{560} t^{3} z_{3} \\
& +\frac{923}{12096} t^{3}-\frac{1}{8} t^{2}+\frac{1933}{43200} t+\frac{61}{1080} t^{3} \lambda_{2}+\frac{1}{1680} t^{7}+\frac{1}{3600} t^{5} \\
& +\frac{1}{288} t^{8} z_{3}+\frac{1}{630} t^{9} z_{3}
\end{aligned}
$$

and

$$
x_{43}(t, z, \lambda)=\frac{2923}{17280} t \lambda_{2}-\frac{1}{4290} t^{13} z_{2}-\frac{1}{240} t^{8} \lambda_{1}+\frac{721}{5760} t^{4} z_{2}-\frac{1}{180} t^{4} \lambda_{1}
$$




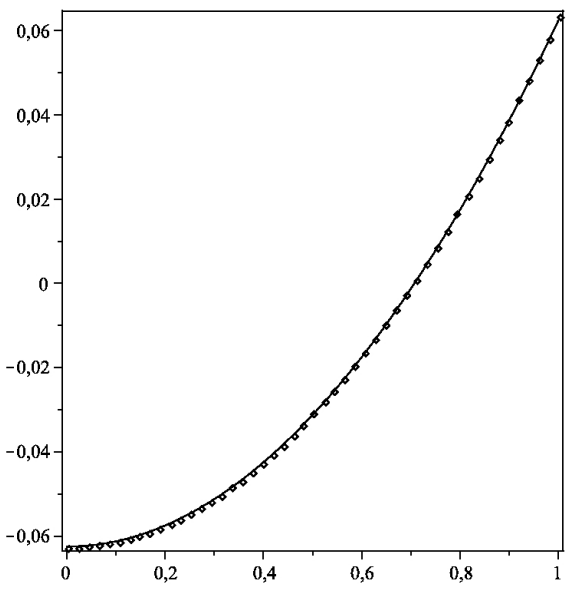

(A) First component

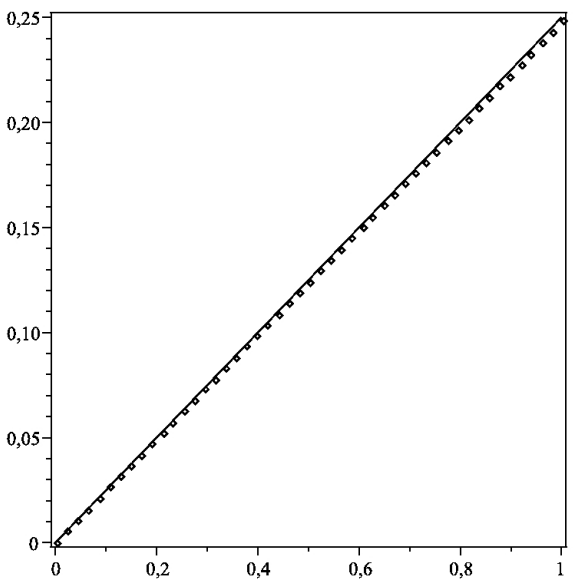

(B) Second component

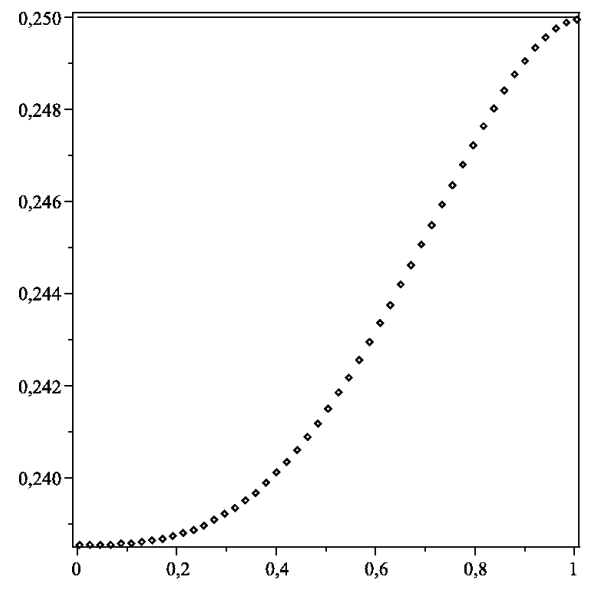

(C) Third component

FIGURE 2. The exact solution (solid line) and its second approximation (dots).

$$
\begin{aligned}
& +\frac{557}{26880} t^{4} z_{3}+\frac{1}{864} t^{9} \lambda_{2}+z_{3}+\frac{1}{480} t^{8} z_{2}-\frac{1}{288} t^{6} \lambda_{2}-\frac{1}{864} t^{6} z_{2} \\
& +\frac{9}{320} t^{6} z_{3}+\frac{1}{15} t^{5} \lambda_{2}-\frac{46}{675} t^{5} z_{2}-\frac{5}{18144} t^{9} z_{2}-\frac{47}{384} t^{4} \lambda_{2} \\
& -\frac{44691}{44800} t z_{3}-\frac{705961}{259459200} z_{2} t+\frac{1}{2800} t^{10} z_{3}+\frac{1}{108} t^{6} \lambda_{1}
\end{aligned}
$$


A CAUCHY-NICOLETTI TYPE PROBLEM FOR EQUATIONS WITH ARGUMENT DEVIATIONS 201

$$
\begin{aligned}
& +\frac{1}{33600} t^{10}+\frac{19}{16128} t^{9}-\frac{1}{11520} t^{8}-\frac{667}{103680} t^{6}-\frac{719}{2160} t \lambda_{1} \\
& +\frac{1}{70} t^{7} z_{3}-\frac{11}{180} t^{5} z_{3}-\frac{3413}{62370} t^{3} z_{2}+\frac{1}{3} t^{3} \lambda_{1}+\frac{1}{945} t^{3} z_{3} \\
& +\frac{101}{15120} t^{3}+\frac{472559}{2073600} t-\frac{1}{9} t^{3} \lambda_{2}-\frac{1}{280} t^{7}-\frac{1}{72} t^{5}+\frac{24869}{967680} t^{4} \\
& -\frac{71}{12096} t^{9} z_{3} .
\end{aligned}
$$

The fourth approximate determining equation has the solution

$$
\begin{aligned}
& \lambda_{1} \approx 0.06249396077, \quad \lambda_{2} \approx 0.2499991352, \\
& z_{2} \approx 0.9764 \cdot 10^{-6}, \quad z_{3} \approx 0.2499875012 .
\end{aligned}
$$

Inserting (10.14) into the formulae above, we obtain the first, second, and third components of the fourth approximation:

$$
\begin{aligned}
x_{41}(t)= & -\frac{1}{16}+0.17635985 \cdot 10^{-3} t^{3}+\frac{1}{8} t^{2}+0.9763918464 \cdot 10^{-6} t \\
& -0.1587242069 \cdot 10^{-3} t^{15}-0.7064456410 \cdot 10^{-8} t^{13} \\
& +0.5050109449 \cdot 10^{-3} t^{11}-0.5284052627 \cdot 10^{-3} t^{7} \\
& -0.124988 \cdot 10^{-5} t^{5}, \\
x_{42}(t)= & 0.9764 \cdot 10^{-6}+0.2034166667 \cdot 10^{-9} t^{10}-0.198393 \cdot 10^{-7} t^{9} \\
& -0.439637 \cdot 10^{-7} t^{8}-0.45884 \cdot 10^{-8} t^{6}+0.479491 \cdot 10^{-5} t^{3} \\
& -0.62494 \cdot 10^{-5} t^{2}+0.2500000001 t+0.13126 \cdot 10^{-6} t^{7} \\
& -0.449797 \cdot 10^{-6} t^{5}, \\
& 0.2499875012+0.1190431552 \cdot 10^{-3} t^{10}+0.72095 \cdot 10^{-7} t^{9} \\
& -0.3471950246 \cdot 10^{-3} t^{8}+ \\
+ & 0.3082364001 \cdot 10^{-3} t^{6}-0.198364 \cdot 10^{-3} t^{3}-0.7 \cdot 10^{-10} t \\
& -0.2275990676 \cdot 10^{-9} t^{13}-0.178554 \cdot 10^{-6} t^{7}+0.639621 \cdot 10^{-6} t^{5} \\
& -0.6613494 \cdot 10^{-4} t^{4} .
\end{aligned}
$$

As is seen from Figures 1-3, the graph of the exact solution almost coincides with those of its approximations (especially with the graph of the fourth approximation). 


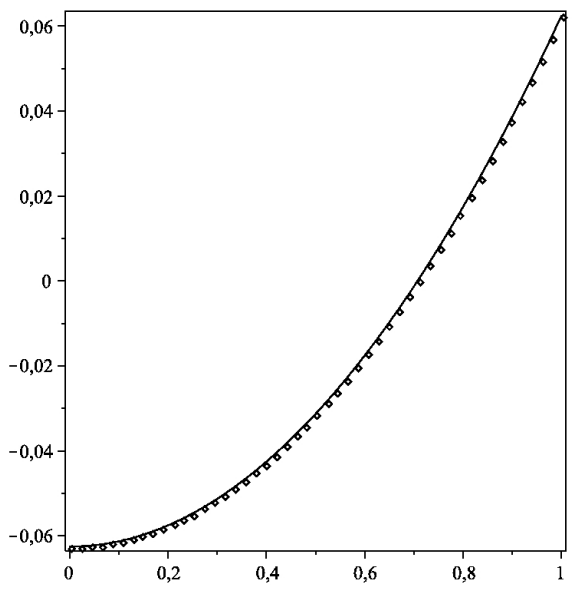

(A) First component

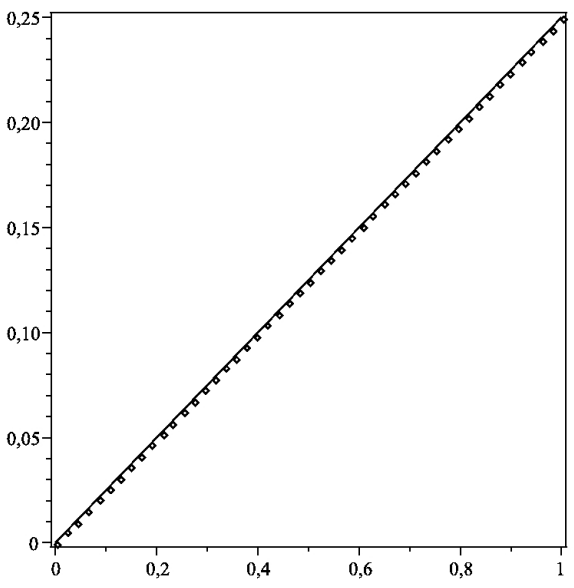

(B) Second component

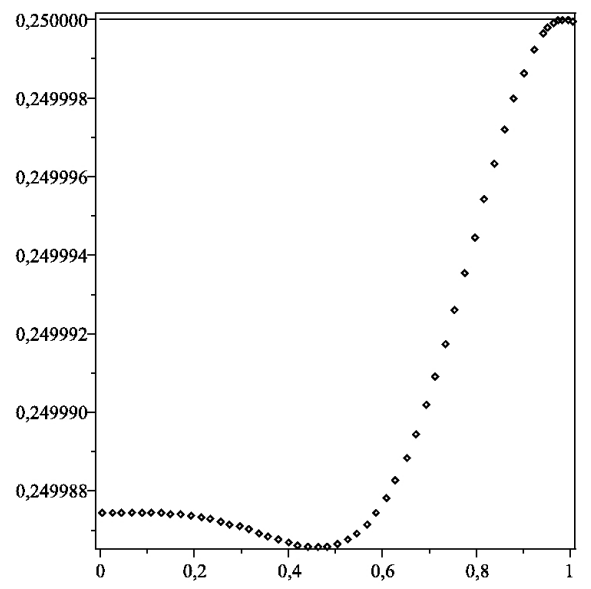

(C) Third component

FIGURE 3. The the exact solution (solid line) and its fourth approximation (dots).

For example, the error of the first approximation (i. e., the uniform deviation of the first approximation from the exact solution) admits the estimates

$$
\begin{aligned}
& \left|x_{1}^{*}(t)-x_{11}(t)\right| \leq 0.8 \cdot 10^{-1}, \\
& \left|x_{2}^{*}(t)-x_{12}(t)\right| \leq 0.14 \cdot 10^{-9}, \\
& \left|x_{3}^{*}(t)-x_{13}(t)\right| \leq 0.25 \cdot 10^{-10},
\end{aligned}
$$




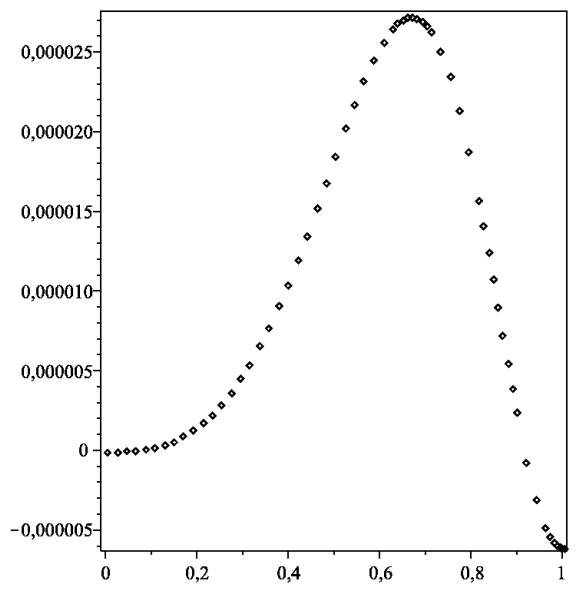

(A) First component

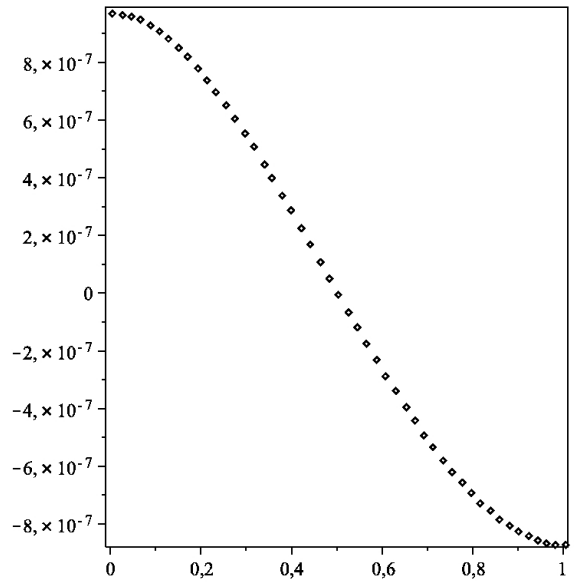

(B) Second component

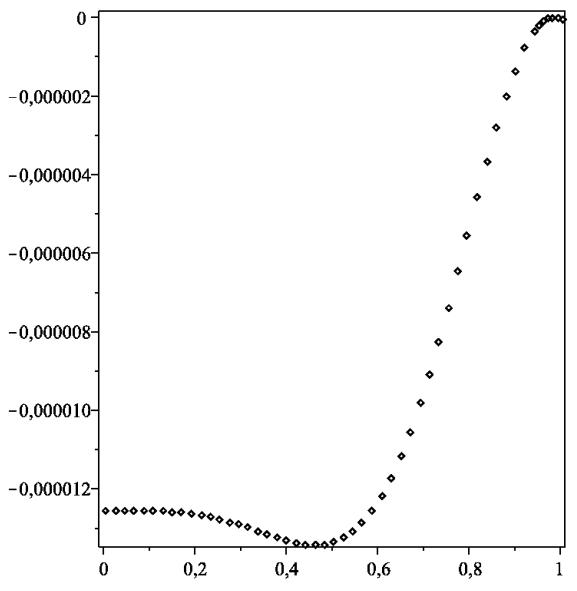

(C) Third component

FIGURE 4. The error of the fourth approximation.

and the errors of the second and fourth approximation are

$$
\begin{aligned}
& \left|x_{1}^{*}(t)-x_{21}(t)\right| \leq 0.12 \cdot 10^{-2}, \\
& \left|x_{2}^{*}(t)-x_{22}(t)\right| \leq 0.8 \cdot 10^{-3}, \\
& \left|x_{3}^{*}(t)-x_{23}(t)\right| \leq 0.12 \cdot 10^{-1}
\end{aligned}
$$


and

$$
\begin{aligned}
& \left|x_{1}^{*}(t)-x_{41}(t)\right| \leq 0.25 \cdot 10^{-4} \\
& \left|x_{2}^{*}(t)-x_{42}(t)\right| \leq 0.1 \cdot 10^{-5} \\
& \left|x_{3}^{*}(t)-x_{43}(t)\right| \leq 0.12 \cdot 10^{-4}
\end{aligned}
$$

\section{REFERENCES}

[1] A. Augustynowicz and M. Kwapisz, "On a numerical-analytic method of solving of boundary value problem for functional-differential equation of neutral type," Math. Nachr., vol. 145, pp. 255-269, 1990. [Online]. Available: http://dx.doi.org/10.1002/mana.19901450120

[2] M. Farkas, Periodic motions, ser. Applied Mathematical Sciences. New York: Springer-Verlag, 1994, vol. 104.

[3] M. Kwapisz, "Some remarks on an integral equation arising in applications of numerical-analytic method of solving of boundary value problems," Ukrainian Math. J., vol. 44, no. 1, pp. 115-119, 1992. [Online]. Available: http://dx.doi.org/10.1007/BF01062634

[4] M. Kwapisz, "On modifications of the integral equation of Samollenko's numerical-analytic method of solving boundary value problems," Math. Nachr., vol. 157, pp. 125-135, 1992.

[5] M. Kwapisz, "On integral equations arising in numerical-analytic method of solving boundary value problems for differential-functional equations," in International Conference on Differential Equations, Vol. 1, 2 (Barcelona, 1991). World Sci. Publ., River Edge, NJ, 1993, pp. 671-677.

[6] A. Ronto and M. Rontó, "A note on the numerical-analytic method for nonlinear two-point boundary-value problems," Nonlinear Oscil., vol. 4, no. 1, pp. 112-128, 2001.

[7] A. Rontó and M. Rontó, "Successive approximation techniques in non-linear boundary value problems for ordinary differential equations," in Handbook of differential equations: ordinary differential equations. Vol. IV, ser. Handb. Differ. Equ. Elsevier/North-Holland, Amsterdam, 2008, pp. 441-592.

[8] A. Rontó and M. Rontó, "Successive approximation method for some linear boundary value problems for differential equations with a special type of argument deviation," Miskolc Math. Notes, vol. 10, no. 1, pp. 69-95, 2009.

[9] A. N. Ronto, "On some boundary value problems for Lipschitz differential equations," Nelīnünn̄ Koliv., no. 1, pp. 74-94, 1998.

[10] A. N. Ronto, M. Rontó, A. M. Samoilenko, and S. I. Trofimchuk, "On periodic solutions of autonomous difference equations," Georgian Math. J., vol. 8, no. 1, pp. 135-164, 2001.

[11] A. N. Ronto, M. Ronto, and N. M. Shchobak, "On the parametrization of three-point nonlinear boundary value problems," Nonlinear Oscil., vol. 7, no. 3, pp. 384-402, 2004.

[12] A. Ronto and M. Rontó, "On the investigation of some boundary value problems with non-linear conditions," Math. Notes (Miskolc), vol. 1, no. 1, pp. 43-55, 2000.

[13] M. Rontó and J. Mészáros, "Some remarks on the convergence of the numerical-analytical method of successive approximations," Ukrainian Math. J., vol. 48, no. 1, pp. 101-107, 1996. [Online]. Available: http://dx.doi.org/10.1007/BF02390987

[14] M. Ronto and A. M. Samoilenko, Numerical-analytic methods in the theory of boundary-value problems. River Edge, NJ: World Scientific Publishing Co. Inc., 2000, with a preface by Yu. A. Mitropolsky and an appendix by the authors and S. I. Trofimchuk.

[15] N. I. Ronto, A. M. Samoilenko, and S. I. Trofimchuk, "The theory of the numerical-analytic method: achievements and new directions of development. I," Ukrainian Math. J., vol. 50, no. 1, pp. 116-135, 1998. [Online]. Available: http://dx.doi.org/10.1007/BF02514693 
[16] N. I. Ronto, A. M. Samoilenko, and S. I. Trofimchuk, "The theory of the numerical-analytic method: achievements and new directions of development. II," Ukrainian Math. J., vol. 50, no. 2, pp. 255-277, 1998. [Online]. Available: http://dx.doi.org/10.1007/BF02513450

[17] N. I. Ronto, A. M. Samoilenko, and S. I. Trofimchuk, "The theory of the numerical-analytic method: achievements and new directions of development. III," Ukrainian Math. J., vol. 50, no. 7, pp. 1091-1114, 1998. [Online]. Available: http://dx.doi.org/10.1007/BF02528821

[18] N. I. Ronto, A. M. Samoilenko, and S. I. Trofimchuk, "The theory of the numerical-analytic method: achievements and new directions of development. IV," Ukrainian Math. J., vol. 50, no. 12, pp. 1888-1907 (1999), 1998. [Online]. Available: http://dx.doi.org/10.1007/BF02514205

[19] N. I. Ronto, A. M. Samoilenko, and S. I. Trofimchuk, "The theory of the numerical-analytic method: achievements and new directions of development. V," Ukrainian Math. J., vol. 51, no. 5, pp. 735-747 (2000), 1999. [Online]. Available: http://dx.doi.org/10.1007/BF02591708

[20] N. I. Ronto, A. M. Samoilenko, and S. I. Trofimchuk, "The theory of the numerical-analytic method: achievements and new directions of development. VI," Ukrainian Math. J., vol. 51, no. 7, pp. 1079-1094 (2000), 1999. [Online]. Available: http://dx.doi.org/10.1007/BF02592043

[21] N. I. Ronto, A. M. Samoilenko, and S. I. Trofimchuk, "The theory of the numerical-analytic method: achievements and new directions of development. VII," Ukrainian Math. J., vol. 51, no. 9, pp. 1399-1418 (2000), 1999. [Online]. Available: http://dx.doi.org/10.1007/BF02593006

[22] A. M. Samoilenko, "A numerical-analytic method for investigation of periodic systems of ordinary differential equations. I," Ukrain. Mat. Zh., vol. 17, no. 4, pp. 82-93, 1965.

[23] A. M. Samoilenko, "A numerical-analytic method for investigation of periodic systems of ordinary differential equations. II," Ukrain. Mat. Zh., vol. 18, no. 2, pp. 50-59, 1966.

[24] A. M. Samoilenko, "On a sequence of polynomials and the radius of convergence of its Abel-Poisson sum,” Ukrainian Math. J., vol. 55, no. 7, pp. 1119-1130, 2003. [Online]. Available: http://dx.doi.org/10.1023/B:UKMA.0000010610.69570.13

[25] A. M. Samoilenko and N. I. Ronto, Numerical-analytic methods of investigation of boundaryvalue problems. Kiev: "Naukova Dumka", 1986, in Russian, with an English summary, edited and with a preface by Yu. A. Mitropolskii.

[26] A. M. Samoilenko and N. I. Ronto, Numerical-analytic methods in the theory of boundary-value problems for ordinary differential equations. Kiev: "Naukova Dumka", 1992, in Russian, edited and with a preface by Yu. A. Mitropolskii.

\title{
Authors' addresses
}

\author{
A. Rontó \\ Institute of Mathematics, Academy of Sciences of the Czech Republic, 22 Žižkova St., 61662 Brno, \\ Czech Republic \\ E-mail address: ronto@math.cas.cz
}

\section{Rontó}

Department of Analysis, University of Miskolc, 3515 Miskolc-Egyetemváros, Hungary

E-mail address: matronto@gold.uni-miskolc.hu 